Programmable Accounting Management for Virtual Private Networks

by

\title{
Anna Evlogimenou
}

A thesis submitted in conformity with the requirements

for the degree of Master of Science

Graduate Department of Computer Science

University of Toronto

() Copyright by Anna Evlogimenou 2001 
Acquisitions and Bibliographic Services

395 Wellington Street Ottawa ON KIA ONA Canada
Bibliothèque nationale du Canada

Acquisitions ef services bibliographiques

395. rue Wellington

Ottawa ON K1A ON4
The author has granted a nonexclusive licence allowing the National Library of Canada to reproduce, loan, distribute or sell copies of this thesis in microform, paper or electronic formats.

The author retains ownership of the copyright in this thesis. Neither the thesis nor substantial extracts from it may be printed or otherwise reproduced without the author's permission.
L'auteur a accordé une licence non exclusive penwettant à la Bibliothèque nationale du Canada de reproduire, prêter, distribuer ou vendre des copies de cette thèse sous la forme de microfiche/film, de reproduction sur papier ou sur format électronique.

L'auteur conserve la propriété du droit d'auteur qui protège cette thèse. $\mathrm{Ni}$ la thèse ni des extraits substantiels de celle-ci ne doivent être imprimés ou autrement reproduits sans son autorisation. 


\title{
Programmable Accounting Management for Virtual Private Networks
}

\author{
by
}

Anna Evlogimenou

\author{
Master of Science, 2001 \\ Graduate Department of Computer Science \\ University of Toronto
}

\begin{abstract}
Until now, proposals for IP accounting management have not exploited the power of programmable networks. Thus, real-time charging and pricing schemes are still regarded as impractical, because the accounting management tasks are executed only at the edges.

In this thesis, we introduce the notion of programmable accounting management which is more flexible, efficient and scalable. Specifically, we propose real-time charging and pricing mechanisms which are practical.

Moreover, we propose a novel programmable accounting architecture for QoS-enabled Virtual Private Networks (VPNs). This architecture gives the flexibility to the providers to employ real-time accounting, and offers an open accounting interface inside the network nodes. This entails that also the VPN subscribers can execute accounting tasks inside the network nodes. For example, they can keep their providers under surveillance, or they can apply their own accounting policies for their users.
\end{abstract}




\section{Contents}

1 Introduction $\quad 1$

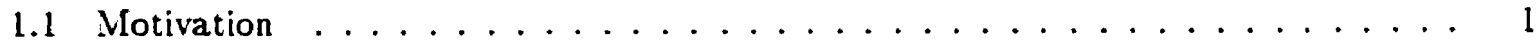

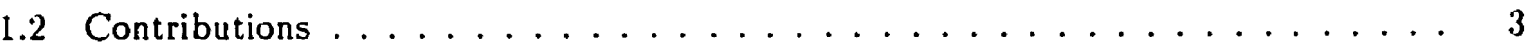

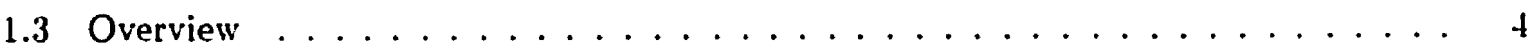

2 Background $\quad 5$

2.1 Programmable Networks . . . . . . . . . . . . . . . j

2.2 Virtual Private Networks . . . . . . . . . . . . . . 6

2.3 Accounting in the Internet $\ldots \ldots \ldots \ldots \ldots$

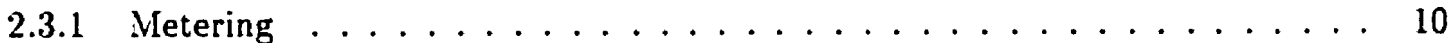

2.3 .2 Charging and Pricing $\ldots \ldots \ldots \ldots \ldots$

2.3.3 Accounting Applications .......................... 15

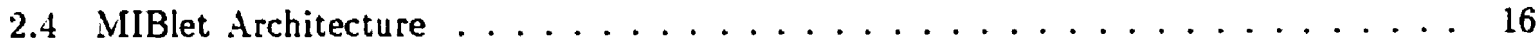

3 Charging and Pricing inside the Programmable Networks 18

3.1 How the Charging and Pricing can be Enhanced by Programmable Networks . . 19

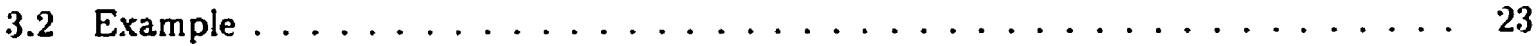

4 A Generic Programmable Accounting Management Architecture for VPNs 28

4.1 Communication Interfaces for Accounting $\ldots \ldots \ldots \ldots \ldots \ldots$

4.1.1 Communication Interface between the AMC/MIBlet Controller and the AM 31

4.1.2 Communication Interface between the AM of the VPN-provider and the AMI of the VPN-customer . . . . . . . . . . . . . . . . 31 
4.1.3 Communication Interface between the AMCs of the VPN-provider and the AMCs of the VPN-customer ..................... 32

4.2 Accounting Components in the Network Node . . . . . . . . . . . . 32

4.2 .1 Accounting Group Controller and MIBlet Controller . . . . . . . . . . 32

4.2 .2 Accounting Management Controllers . . . . . . . . . . . 33

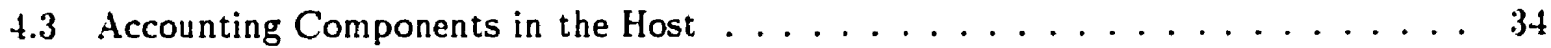

4.4 Accounting Manager . . . . . . . . . . . . . . . . 34

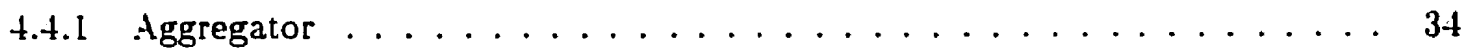

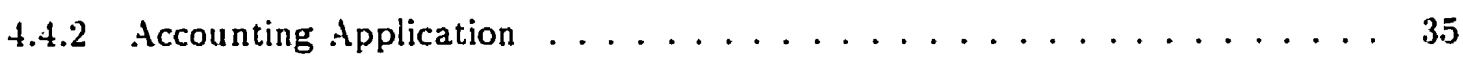

4.5 Discussion of Having A.MCs Both at Hosts and Network Nodes . . . . . . . 35

5 Case Study $\quad 37$

5.1 Charging and Pricing Techniques ................... 40

5.2 Metering, Accounting Protocol and Billing .............. 43

5.2 .1 Functional Blocks of Accounting Management . . . . . . . . . . 43

5.2.2 Messages Exchanged among the Functional Blocks . . . . . . . . . 49

5.3 Further Extensions of the Proposed Example . . . . . . . . . . . . 54

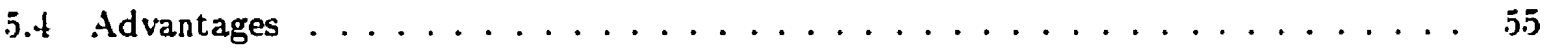

6 Conclusions $\quad 57$

6.1 Related Work . . . . . . . . . . . . . . . . . 57

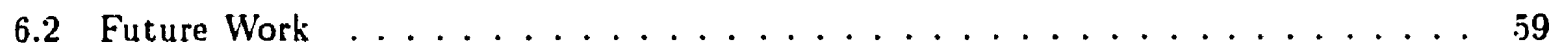

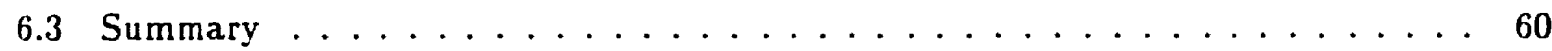




\section{List of Figures}

1.1 Accounting management for VPN networks, where the VPN provider sells VPN services and the subscribers who buy VPN service may retail IP services to their

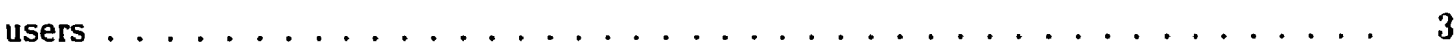

2.1 Interactions of the components of accounting management $\ldots \ldots \ldots$

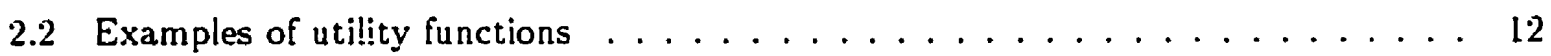

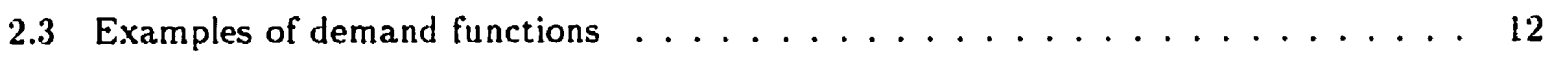

2.4 MIBlet architecture: There are two VPN customers: A, B . . . . . . . . . 17

3.1 Customer's mechanisms inside the network node $\ldots \ldots \ldots$

3.2 Traditional smart market scheme vs. Smart-market in programmable networks . . 22

3.3 A charging and a pricing model executed in the host at time slot $k \ldots \ldots 24$

3.4 A charging and a pricing model executed in the network node at time slot $k \ldots 25$

3.5 Algorithm that user $r$ executes in the network node $\ldots \ldots \ldots$

4.1 Overall Accounting Management architecture in a customer VPN, called VPN A . 30

4.2 Accounting Components in a network node $\ldots \ldots \ldots \ldots$

4.3 Architecture of Accounting Manager . . . . . . . . . . . . 35

5.1 A VPN Customer with two sites: Site A and Site B; customer requests VPN Service from its two Local VPN Providers: Provider A and Provider B . . . . . . . . 37

5.2 Provider B functions, which are performed at Network Management Layer . . . . 41

5.3 Customer function for buying SLAs from the provider $B$, which are performed at Network Management Layer . . . . . . . . . . . . . . . . . . . . 42 
5.4 Customer functions for buying resources from the provider $A$, which are performed at Resource Management Layer . . . . . . . . . . . . . . . . . 43

5.5 Provider A functions, which are performed at Resource Management Layer . . . 44

5.6 Functional Blocks in the Accounting Manager of Provider A and B. In Provider A there is not any "Pricing" component, since the charging and pricing is performed in the Resource Management Layer . . . . . . . . . . . . . . . . . . . 46

5.7 Functional Blocks in the Network Management Layer . . . . . . . . . . . ti

5.8 Functional blocks in the Resource Management Layer of Provider A. In Provider $B$ the Bidding Controller does not exist and instead of having an auction-based scheduler, there is a simple scheduler . . . . . . . . . . . . . . . . 49

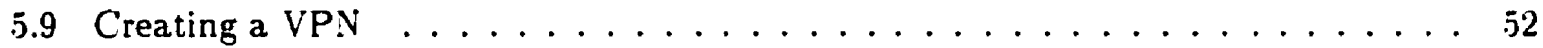

5.10 Charging and Pricing in the Resource Management Layer of Provider A . . . . 53

5.11 Charging and Pricing in the Network Management Layer of Provider B . . . . . 5.3

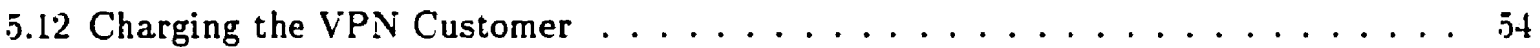




\section{Chapter 1}

\section{Introduction}

A Virtual Private Network (VPN) that exists over a public infrastructure, like the Internet, is both cheaper and more flexible than the network which is composed of leased lines. A major concern of providing VPN services over the Internet is to segregate VPN's traffic from the other traffics which share the same network infrastructure. Nowadays, there are Internet protocols which can satisfy the need of data integrity and privacy. Another concern is that VPN subscribers require to ability to control and manage "their" network resources, in order to run service specific control software (e.g., run a protocol tuned to support the requirements of video conferencing). Programmable Virtual Private Networks have been advocated as a means of addressing this issue.

In this thesis, we examine how the programmable network can enhance the accounting management, and then we propose an accounting architecture for programmable VPNs. Accounting management involves the following operations: metering, charging, pricing and billing.

\subsection{Motivation}

The real-time accounting management is still an open issue. There have been proposed several schemes, but few of them have been implemented and tested in a real environment. All of these schemes assume that they can be executed either at the end-points of the network (i.e, hosts), or at the accounting server. However, some input parameters of these models exist only inside the network. Thus, they require feedback signals from the network nodes in order to perform their operations. We can decrease the traffic overhead induced by these tasks if we execute them inside the network nodes. 
The principle of transferring tasks to the network nodes is actually followed in the programmable networking philosophy. In other words, the intelligence is pushed to the core of the network instead of leaving at the end-points. An accounting management scheme over a programmable network will offer: (a) real-time metering, charging and pricing, (b) distributed management which increases scalability and performance, and (c) integration of new mechanisms for charging and pricing without the need to introduce new protocols. These are the main motivations which lead us to consider the programmable networks for executing the accounting management tasks inside the network nodes instead of at the end-points or at the accounting servers. We should note that Travostino used the programmable networks for processing the metering data at the points from which they are derived (i.e., network nodes)[1].

In addition to the above motivation, accounting management for the VP. networks is an important task. For instance, a VPN provider allocates a portion of its resources to the subscribers. i.e., it provides some level of qualities of services (QoSs). However, QoSs derive the need to encourage the subscribers to select the service that is most appropriate for their needs and to discourage them from always requesting the highest QoS or over-allocating the resources. This is accomplished through the accounting management. Specifically, resource allocation is translated into monetary units, and requesting more resources is straightforwardly reflected in the amount of money the subscriber is charged (i.e., the subscriber has to pay more money).

The main difference between accounting management in traditional networks and accounting management in programmable VPNs is that the VPN subscribers request real-time access to their accounting information, and also wish to monitor and control effectively their traffic. Figure 1.1 illustrates the ISP 1 and ISP $\mathrm{n}$ which sell VPN services. In turn, their VPN subscribers may retail IP services to their users. The arrows represent the flow of money. If subscribers retail IP services, then they must monitor and control their users' traffic, and apply their own accounting management schemes, for charging their users. This entails the need of developing an open accounting interface for letting subscribers apply their accounting policies in critical network nodes.

Another concern of accounting management for VPNs is that VPNs span many Internet Service Providers (ISPs). Each ISP is expected to deploy its own charging and pricing policies. Thus, a VPN subscriber has to deal with heterogeneous policies, whereas the subscriber who asks for a simple IP service has to pay only his local ISP. In order to handle this heterogeneity, the VPN 


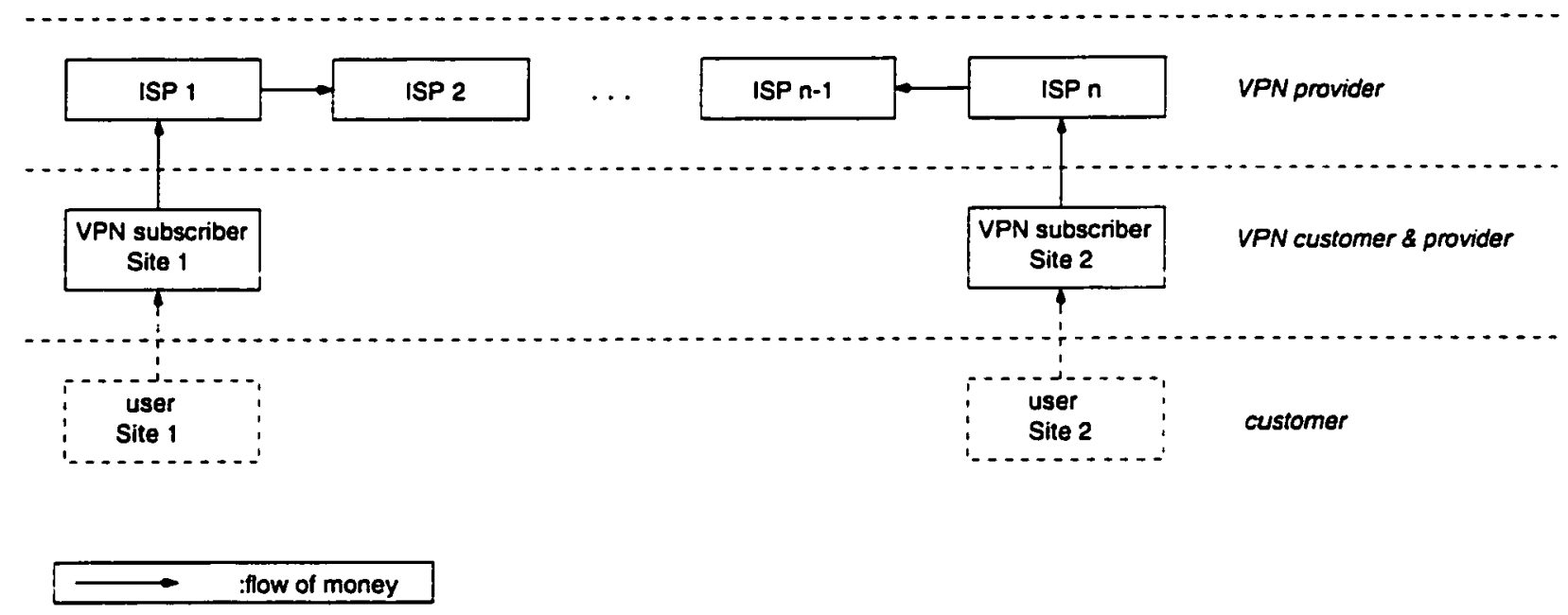

Figure 1.1: Accounting management for VPN networks. where the VPN provider sells VPN services and the subscribers who buy VPN service may retail IP services to their users

subscriber has to coordinate its resource requirements among the ISPs. and also he may have to map the services provided by the one ISP into the services provided by the other ISPs.

Although it is emphasized that accounting management is of great importance in programmable VPNs, there is no work which proposes an accounting architecture for VPN networks. In addition, to our knowledge, there in no work which examines the issue of charging and pricing in the context of programmable networks.

\subsection{Contributions}

The contributions of this thesis are:

- Enhancing the charging and pricing mechanisms by exploiting the programmable networks: There are some problems with the traditional real-time pricing and charging models which can be solved by executing them inside the network nodes. These problems are specified. In addition, we give an example of migrating a specific pricing model from the end-points to the network nodes.

- Proposing an accounting management architecture for programmable VPNs: A programmable accounting management architecture for VPNs is described. Furthermore, we demonstrate our architecture with specific charging and pricing algorithms. 


\subsection{Overview}

The thesis starts with a survey of programmable networis, VPNs, and accounting management in Chapter 2. Chapter 3 examines how the programmable networks can enhance the charging and pricing models. In Chapter 4, we present our accounting management architecture. Then, Chapter 5 presents a concrete example which exploits the main characteristics of our architecture. Finally, Chapter 6 presents related work and concludes this thesis. 


\section{Chapter 2}

\section{Background}

In this chapter, we provide the background of this thesis. First. we define the programmable networks and the two basic directions of this research area. Then, we present methods for providing Virtual Private Networks (VPNs), and the open issues of VPNs. In the third section, the literature related to accounting management is surveyed. Finally, we present the MIBlet mechanism, which is used as a basic mechanism in Chapter 4.

\subsection{Programmable Networks}

The goal of programmable networking [2] is to facilitate the deployment of new network services, and the customization of the existing network services by providers and not only by manufacturers. Thus, the time-to-market for the providers can be greatly reduced. A representative example is the demand to add quality of services to the Internet. Although Integrated Services $[3,4,5]$ and Differentiated Services $[6,7,8]$ have been standardized, the current Internet still does not provide these services. If the network nodes were programmable, then these services could be provided faster.

However, the separation of the role of equipment vendors from that of software and service providers poses some challenging issues: how programmable network interfaces should be defined: how much abstraction is called for; new cost models [9]; performance, robustness and security issues. There are two directions of the research in programmable networks represented by the Open Signaling [10] and Active Networks [11].

The Opensig working group provides open access to switches and routers using a set of open 
programmable network interfaces. Opensig is primarily focused on programmable switches that provide some level of QoS, and takes a telecommunication approach to the problem where there is a clear distinction between transport, control and management. The IEEE Project 1520 tries to standardize the programming interfaces for ATM switches, IP routers and mobile telecommunication networks.

On the other side, Active Networks provide more dynamic network programming interfaces than Opensig. Active Networks allow the customization of network services at packet transport granularity rather than at the programmable control plane. An example of Active Networks, is “active packets" or "capsules" which comprise executable programs, consisting of code and data. However, Active Networks offer maximum flexibility in service creation at the expense of more complex programming model. Finally, this community is focused on IP networks where the control and data paths are combined.

\subsection{Virtual Private Networks}

Ferguson and Huston define a Virtual Private Network (VPN) as: "A communication environment in which access is controlled to permit peer connections only within a defined community of interest, and is constructed though some form of partitioning of a common underlying communications medium, where this underlying communications medium provides services to the network on a non-exclusive basis"[12]. Lintil now, VPNs have been most commonly requested to connect corporate LANs, but it is expected that in the future a VPN can be created for just the duration of a video conference, and individual users will be able to ask for a VPN service. Today, VPNs are deployed on a public network infrastructure, the Internet. There have been proposed many VPN solutions, which can be categorized into two major models: overlay VPN models, and peer VPN models. In the following, we describe briefly these two models, and then we present the future trends in VPNs.

In the overlay model, the router in a VPN site connects via point-to-point links to routers in other sites. The intermediate link layer network is used as a "cut-through" among the VPN sites. The technology that is used for the overlay model is Frame Relay, leased lines, or ATM circuits. The VPN solutions based on this model are predominant nowadays, though this model has scalability problems. Specifically, when a VPN customer has a relatively large number of sites, 
the amount of routing information maintained for each router in VPN sites becomes very large. If the number of sites is $N$, then each router needs $(N-1)$ routing peerings in order to have full mesh connectivity among these sites. Another problem is the number of configuration changes required when adding a new site to an existing VPN. Every router must be updated.

Alternatively, in the peer model, the routers in VPN sites interacts directly with the provider routers, and the routers in the intermediate transit path interact with the next-hop routers. In this way, many disadvantages of the overlay model are overcome. By deploying a VPN solution based on the peer model, a VPN provider can support thousands to millions VPNs. and VPNs which consist of hundreds or even thousands of sites. An example of the peer model is "Controlled Route Leaking", where the routes for a VPN customer are filtered and they are not announced to any other part of the network, and non-VPN routes are not all announced to the VPN customer. However, this solution is prone to producing erroneous configurations due to human errors. BGP/MPLS VPN [13] is a solution based on the BGP protocol, on multiple forwarding tables, on usage of a new type of addresses (VPN-IP addresses), and on MPLS technologies. This solution alleviates the problems of the other solutions.

All the above VPN solutions have been focused on techniques for segregating the traffic originated by VPNs. However, issues of network performance, quality of services and mechanisms for charging and pricing are still open, and they are of great importance. These issues have been addressed in the research area of programmable VPNs. Programmability gives the flexibility to VPN customers to manage and control the physical network resources. For example, customers can deploy low-level monitoring of their traffic, or they can control its routing and addressing mechanisms. The difficulty of providing programmable VPNs, is that the provider should isolate one VPN from another, so that each customer can have exclusive control and access to "his" resources.

In the project VNRMS [14], a new class of programmable networks was introduced. Specifically, many VPNs can be generated out of a single physical network node. VPNs can be spawned and combined recursively to create multiple layers of VPNs. Each spawned VPN acts as a client of the parent-VPN, and it can control and manage "its" resources. The parent-VPN acts as a server. Similarly, in Genesis [15], a network environment which is capable of dynamically creating new network architectures on-the-fly has been designed and implemented. The life cycle of creation, deployment and management of VPNs is automated. It is similar to an operating system which 
spawns a child process, i.e., it treats a copy of the calling process. The child process inherits its parent's attributes.

To our knowledge, there is not any work which addresses the issue of Accounting Management for Programmable VPNs. Though, it is emphasized that accounting management is necessary for providing programmable VPN services, in the literature there is not any proposed architecture for accounting.

\subsection{Accounting in the Internet}

The Internet provides a best-effort service. However, the demand for deploying QoSs implies also the need for accounting management in the Internet. QoSs and accounting management encourage the users to choose the service that is most appropriate for them, thereby discouraging over-allocation of resources and abuse of network resources. Furthermore, public subsidies for the Internet decrease and in the near future will cease. Thus, another reason that the providers have to adopt pricing models for charging their customers is to maintain and replace their out-of-date equipment.

In the literature, the terminology used for Internet accounting is quite confusing. In this thesis, the term accounting management is used to denote the four processes: metering. charging, pricing, and accounting applications. Note that the research community has adopted the "divideand-conquer" approach for the accounting management by dividing it into many research areas. However, there is close relationship among these areas. Figure 2.1 depicts the interaction of the four steps of the accounting management.

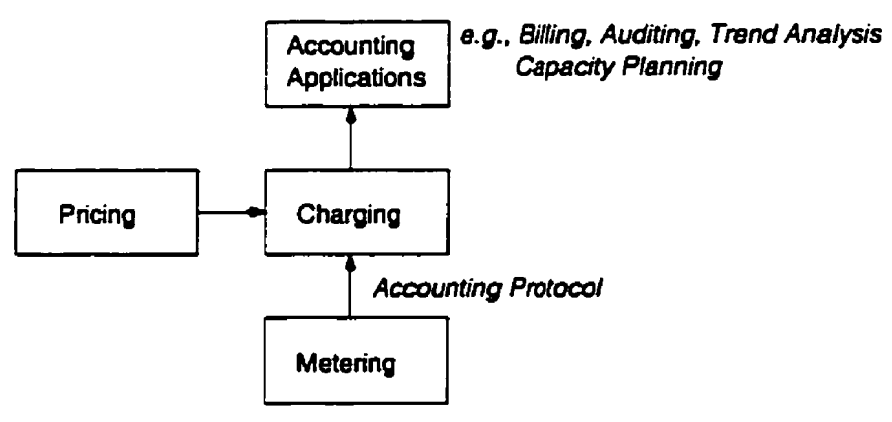

Figure 2.1: Interactions of the components of accounting management

In one of the first research papers on accounting [16], Shenker et al. criticize existing research 
on optimal pricing policies and suggest that the research agenda should focus more on structural and architectural issues. They propose the edge-pricing model where the services are charged at the edge, i.e., at the local ISP. In the same way, ISPs purchase service from each other. Adjacent ISPs exchange bilateral agreements, and each ISP takes the full responsibility for every packet it forwards. This architecture allows each ISP to employ its own pricing policies. Finally, Shenker et al. reveal two major problems in accounting architectures: (1) charging appropriately for multicast, and (2) the ability to charge receivers for the service. The first problem is that the multicast address is a logical name and does not reveal any geographic or provider information. Moreover, the members of the multicast group can change quite rapidly and so the mechanisms for providing the appropriate accounting information must be designed with care. The second problem involves the following issues: (1) how does the receiver indicate to the network provider the willingness to take the responsibility for the source's traffic? (2) how does the network "bill" the receiver? (3) how does the network split the responsibility for the bill among the members of a multicast group?

Another preliminary work which addresses the architectural issues of pricing is done by Clark [17]. He proposed a scheme where users are charged only by their local ISP. More specifically he proposed the expected capacity model which later was expanded into the Differentiated Services (DiffServ) model. Finally, he points out the following limitations of his scheme: (1) transitive capacity problem: if a traffic stream crosses a number of providers from the source to the destination, then ideally all providers should purchase enough capacity from their adjacent providers. But what if this does not happen? There could be a scenario where a provider cannot afford to buy more capacity or most commonly the receiver may have a final access link that represents a bottleneck. In this situation, a sender pays for a specific amount of expected capacity, while receiving congestion signals. (2) Sender pays; receiver pays: in other words the receiver cannot select a service at the local ISP of the sender and the receiver cannot show that he is the one who will pay for the service at the sender's local ISP.

In the sequel, we describe briefly each of these processes and we address the most important works. Note, that metering is not necessary when flat rates are adopted by ISPs. However. metering is required for usage-based charging schemes. 


\subsubsection{Metering}

The first process of accounting management involves monitoring and measuring the network resources. Metering granularity varies depending on the network layer at which the meter is employed. One of the first works in metering was done in the University of Waikato of New Zealand [18]. Since 1990 the Internet gateway of New Zealand universities monitored and charged its users by volume. The reason was that the New Zealand government did not subsidize this gateway. This experience concludes in 1995 that the overheads of traffic monitoring are significant.

Efforts for standardization of mechanisms and architectures have been made by the RealTime Traffic Flow .Measurement (RTFM) working group of IETF. In addition, NeTraMet [19] is the first implementation of the Internet Accounting Architecture $[20,21]$ and is used by many Internet Service Providers (ISPs).

An accounting protocol is responsible for transmitting the metering data from the network resources to the accounting manager. Considering proposals for accounting protocols, IETF has established the Authentication, Authorization and Accounting (AAA) Working Group. However, IETF has not proposed a new protocol, but rather proposes a piggy-backed AAA protocol. For that purpose, Mitton et al. [22] compare the Remote Access Dial-In User Service++ (RADIUS++), Simple Network Management Protocol (SNMP), DIAMETER and COPS protocols. The most suitable solution is DIAMETER and then the COPS according to the authors.

An issue of traditional meter design is that it does not consider processing traffic data inside the meter, but rather it transmits raw data at the cost of network overhead.

\subsubsection{Charging and Pricing}

In the second step (see Figure 2.1), which composes the core of the accounting management, there are two processes: charging and pricing. In the charging process, the tariffs and the accounting data, from the second step, are combined to charge the users. Therefore, through this process the accounting records are transformed into monetary units for users over some period of time. Tariffs are determined by the pricing process.

Before we examine the charging and pricing techniques, we should answer the question: what are the costs of providing bandwidth? Mackie-Mason and Varian group these costs into three groups [23]: fixed costs, incremental costs or congestion costs, and cost of capacity expansion. The 
infrastructure costs, costs to access lines and switching equipment, and salary costs compose the fixed costs. These fees will be different for different people since different people and institutions will value the connection to the net differently. The incremental cost of sending extra packets depends on the network load. If there is no congestion, then this cost is essentially zero because no additional real resources are used. On the other hand, when the network is congested then this cost is positive, because if the packet is transmitted, then it will cause delay and dropped packets to the other users. Thus, this cost should reflect the marginal cost of delay from an economic point of view. Finally, the expansion of network capacity is useful when congestion occurs. This cost reflects the marginal benefit of transmitting one more packet.

One of the debates in Internet pricing is whether flat rates or usage-sensitive fees should be charged to users [24]. The only advantages of flat fees are that the implementation is easy, and that there is little overhead for billing. On the other hand, flat rates cannot cover the congestion costs, are unfair for light users, are not appropriate for differentiated QoSs, and do not discourage the misbehaving users. However, usage-sensitive pricing covers the congestion costs and it increases the fairness. The disadvantages of this type of pricing are: it may discourage usage; the implementation is complex; and it is difficult for users to budget for a network expense that is uncertain. The Internet Demand Experiment (INDEX) [25], conducted at University of California at Berkeley, tried to characterize the customer behaviour in relation to network services. They built an Internet Service Provider for residential users, which offered differentiated services. There were many quality/price combinations. The results showed the inefficiencies of the current flat-rate model, and the benefits from the usage-based pricing for both users and provider.

In the rest of this section, we define the basic terms and the objectives of usage-based pricing, and then we classify the models that have been proposed until now. The common terms which are used in Internet economics are:

- Utility function: in usage-based pricing models, the user's willingness to pay for specific QoS is modeled by the utility function. Utility is expressed as a function of QoS parameters, such as delay or packet loss. However, in real networks it is impossible to predict in advance such quality measures. Therefore the utility is expressed as a function of resources made available to a user. Also, there is a debate in Internet pricing whether the utility functions can be estimated or not. Shenker et al. argue that the relationship between handling individual packets and the overall utility is extremely complex [16]. Some examples of utility functions 
are depicted in Figure 2.2 [24].
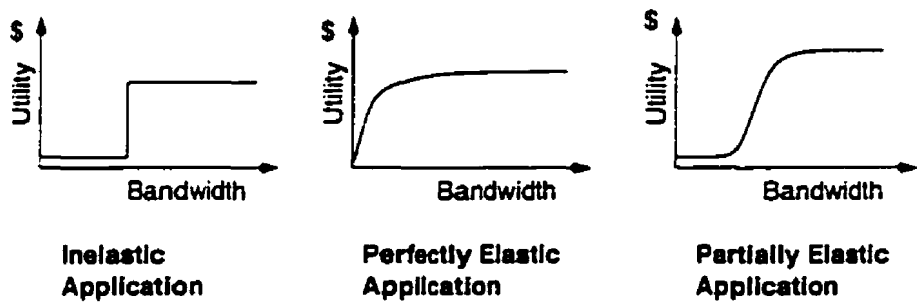

Figure 2.2: Examples of utility functions

- Customer surplus (or user benefit): customer surplus is defined as the difference between the maximum amount a customer is willing to pay for a given service and the amount he is charged for it. In other words, customer surplus is the difference between utility and price.

- Demand curve: The user's demand is modeled as a function $D(p)$, where $p$ is the price per unit of access (such as bandwidth). The meaning of the demand curve is that each of the $D(p)$ units of access is worth at least $p$ to the user, and every additional unit is worth less than $p$. The equation which relates the utility with the user's demand is: $u(x)=\int_{0}^{x} G(x) d x$. where $u()$ is the utility function, $G()$ is the inverse demand function of user. In Figure 2.3 two examples of demand functions are depicted.
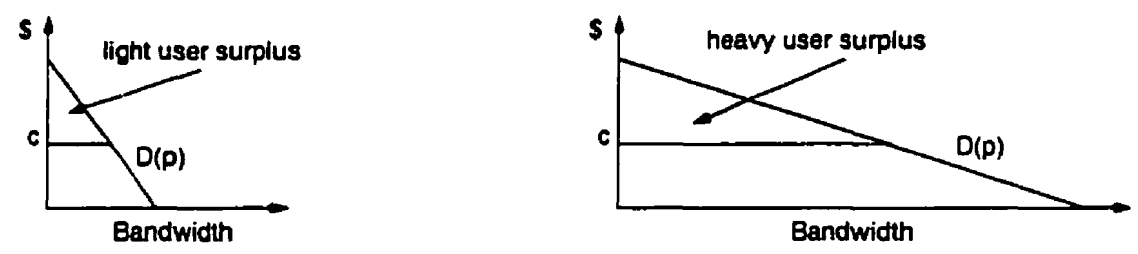

c: cost per unit of bandwith

Figure 2.3: Examples of demand functions

- Producer surplus: Producer surplus is the difference between the amount that customers are charged for a service and the cost of providing this service.

Setting prices is often treated as an optimization problem with various possible objective functions. We classify these objective functions into two categories: maximize the social welfare; maximize the producer surplus. In the former class, the Internet is viewed as a public good, in 
which benevolent service providers set the prices in order to maximize the social welfare (i.e., the consumer surplus). The costs in this class are called marginal costs. Whether these marginal costs can cover the fixed costs or not is another controversial topic in pricing communication networks [16]. In the latter class, the Internet is viewed as a private good and the service provider tries to maximize his revenue.

In this paragraph, we discuss the usage-based pricing schemes that have been proposed in the recent literature [26]. These are:

- Paris-Metro Pricing (PMP) [2i]: This proposal was based on the pricing of the ParisMetro. Briefly, links are divided into separate logical channels whose bandwidth is fixed. The prices of the links remain constant for extended periods. This scheme does not provide guarantees of quality of service. However, the channels with higher prices would be less congested than those with lower prices. The advantage of this approach is that it is very close to the flat-rate approach which attracts users since it is simple and prediclable.

- Priority Pricing: Pricing schemes based on priority pricing have been proposed [28, 29]. Priorities, still, do not provide guarantees of QoSs. Obviously, higher priorities cost more than lower priorities. Prices change on short time scales (on the order of minutes). Gupta et al. suggest that providers set the prices of priorities according to network load with the goal of preventing the congestion [28]. The authors show through simulations that this pricing scheme improves the service level. On the contrary, Marbach suggests having the costs of priorities change on long time scales [29]. Users mark their packets with a priority in order to respond to the network load. Thus, when there is congestion, users choose higher priorities than during the off-peak periods.

- Smart-Market Pricing: This scheme was proposed by economists Mackie-Mason and Varian and is perhaps is the most influential proposal in Internet economics. It is based on auctioning [23]. The user associates a bid with each packet. which denotes the user's willingness to pay for transmission. The network node sorts all the bids; determines the threshold value, which is the marginal congestion cost; and finally the packets whose bid is greater than this marginal cost are transmitted and are charged this cost. Roughly, all the transmitted packets are charged less than their bids, and consumer's surplus is always positive. Furthermore, with this scheme the network benefit is always maximized. Similarly 
with the previously pricing schemes, no guaranteed QoSs are provided. However there are problems with the auction-based schemes: (1) the bidding process should be carried on a hop-by-hop manner, but the users do not have the knowledge of which links inside the network are congested and they only care whether their packets reach the final destination, (2) when a packet is not transmitted because its bid was low, it is buffered, and the delay can be predicted neither by the user nor by the provider. The problems of this scheme have induced many criticisms of this pricing technique in the network community. Recently, Wackie-Mason has proposed a smart-market pricing model for reserving resources in multiple quality of service networks [30].

- Expected Pricing: Shenker suggests a charging scheme based on two approximations: the expected congestion and the expected path [16]. Thus, prices are set according to the time-of-day and not to the congestion level. He also points out that there is a disadvantage with this approach: the inability to charge less during periods of low congestion. Moreover he suggests using the expected path, where the charge depends only on the source and destination, and not on the actual path. Furthermore, Clark proposes a resource allocation and pricing scheme based on expected capacity [17]. Expected capacity only indicates the behaviour during the congested periods, and does not restrict a user to send packets when the network is under loaded. An advantage of the expected pricing approach is that there is no need for measuring.

- Responsive Pricing: Mackie-Mason, Murphy and Murphy propose a scheme based on feedback signals [31]. More specifically, users adapt their traffic input according to the pricing feedback. The prices should vary dynamicalty based on the current utilization of network resources. Thus, when prices increase, users transmit fewer packets in the next time interval. There are two classes of adaptive users: elastic users who cannot tolerate loss but they can await feedback from the network that indicates when they can input traffic; and inelastic users who require a delay guarantee, but can tolerate loss. Simulation results show that both network efficiency and economic efficiency increase. This scheme was suggested for the ATM ABR class of service, where users who respond "appropriately" to dynamic feedback get loss guarantees from the ATM network.

- Effective Bandwidth Pricing: The concept of effective bandwidth is used to provide a 
measure of resource usage of sources with varying characteristics and requirements. For general models of sources and resources it is possible to associate an effective bandwidth with each source such that, provided the sum of the effective bandwidths of these sources is less than a certain level, then the resource can deliver a performance guarantee. The pricing schemes proposed in this category can support guarantee of QoSs, and require Call Acceptance Control (CAC). Kelly proposes a charging scheme based on effective bandwidth [32] . The tariff is a linear function of: a charge $\alpha(x)$ per unit time, a charge $b(x)$ per unit volume of traffic carried, and a charge $c(x)$ per connection, where the triple $\{\alpha(x), b(x), c(x)\}$ is fixed at the time of connection $x$ ( $x$ denotes the traffic characteristics). Thus the cost of a connection is: $\alpha(x) T+b(x) V+c(x)$, where $T$ is the duration of the connection and $V$ is the volume of the connection. The most important feature of kelly's scheme is that the static parameters and dynamic parameters are distinct. The $x$ which represents the static parameters is constant during the connection, whereas the $T, V$ are measured in real-time. Courcoubetis and Siris propose a scheme for pricing SLAs (Service Level Agreements) for differentiated service that is based on an upper bound of the effective bandwidth [33]. The network publishes a family of pricing curves that reflect the traffic load. Then, each user chooses a traffic contract that includes information on the traffic characteristics (peak rate and token bucket parameters). Prices change on long time scales and are supposed to be constant for the duration of the SLA.

\subsubsection{Accounting Applications}

At the final step, accounting applications such as billing, auditing, capacity planning and trend analysis are performed. Each application does not have the same security and reliability requirements [34]. As a consequence, the goal of [ETF is to provide a set of tools that can be used to meet the requirements of each application, rather than designing a single accounting protocol and a set of security services that will meet all the needs. The purpose of each application and its requirements are:

- Billing: Billing involves consolidation of accounting records per customer and issuing of invoices. The web based interface combined with Java can automate the customer to service provider as well as the service provider to service provider interactions [35]. It can also reduce the costs of both sides. Payments can be made either inband or outband [36]. Outband 
payments are implemented by means of credit cards, bank transfers and checks, whereas inband payments are implemented in terms of cybercash. Billing applications are concerned with reliable data collection and security; otherwise, packet loss or fraud may lead to revenue loss.

- Trend analysis and capacity planning: The goal of these applications is to forecast the future usage. Thus, these applications can tolerate packet losses, and security requirements are desirable when data crosses administrative domains.

- Auditing: Auditing tasks include verifying the correctness of an invoice submitted by the service provider, or verifying conformance to usage policing, service agreements, or security guidelines. Auditing should be at least as reliable as the accounting process, and security service may be required.

\subsection{MIBlet Architecture}

Each network node contains a Management Information Base (MIB) which stores information about configuration, statistics and status of resources of the network node. Through the MIB, we can monitor and control the resources of the network node. A MIBlet [3T] is a logical partition of the MIB. Each customer has his own MIBlet controller and he can control and manage his resources through the MIBlet. Network resources of each customer are segregated through the MIBlet controllers.

The MIBlet concept can be applied straightforwardly to VPN design. control and management. We will describe briefly how this can be achieved. First of all, there are two layers (see Figure 2.4): Network Management Layer and Resource Management Layer.

In the Network Management Layer, the VPN provider manages and control its resources through the MIB, and the customers manage and control their resources through the MIBlets. Furthermore, in this layer the customers can request a VPN service. they can reconfigure their topology, or they can ask for termination of their VPN service.

In the Resource Management Layer, there are the network nodes. These nodes are assumed to be programmable. Thus, MIBlet controllers can be executed inside the network nodes. The VPN provider installs the MIBlet controllers in the network nodes when a customer requests a VPN service. When the provider has successfully created all the MIBlet controllers, then the customer 


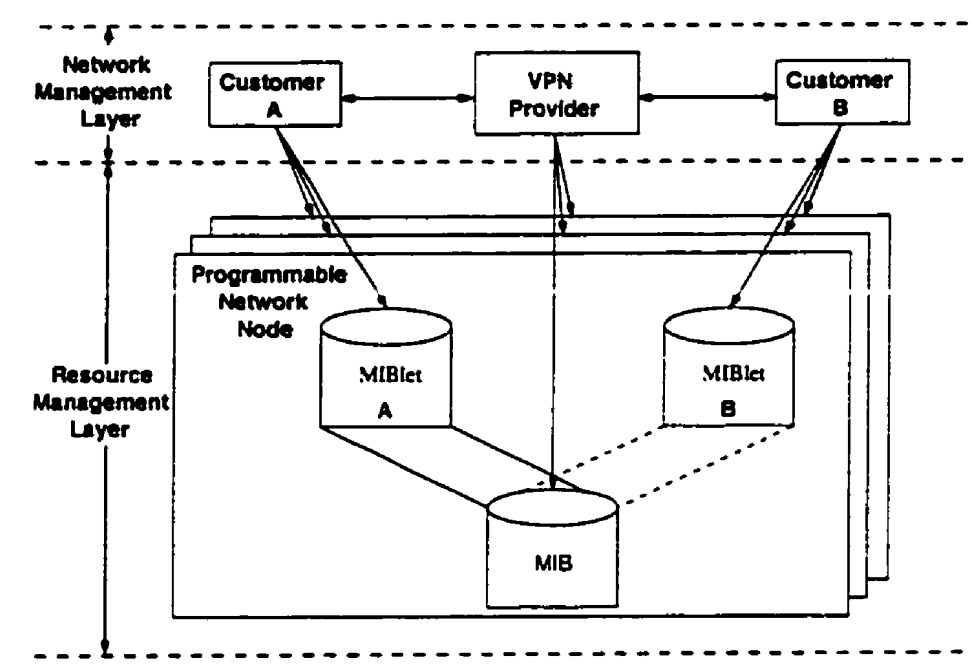

Figure 2.4: MIBlet architecture: There are two VPN customers: A. B

can communicate directly with them, using a standard protocol. such as S.MMP. 


\section{Chapter 3}

\section{Charging and Pricing inside the Programmable Networks}

In this chapter, we propose a new charging and pricing mechanism which executes inside the network nodes. In this way, common problems can be overcome at the expense of requiring more CPU cycles. Then we show the benefits of our mechanism by presenting an example. Before we discuss our mechanism, we explain the term of charging granularity.

The charging granularity is determined by the pricing policies. The pricing policies can be either dynamir or static. If dynamic pricing is applied, then the costs fluctuate according to the network load, and the charging granularity is on a per-packet level. An example of dynamic pricing is the auctioning, which actually is argued to be an optimal pricing model. On the other hand, if pricing is static, the costs are independent of the network conditions. For instance, when the costs of SLAs are determined by the time of the day, then the pricing is static.

The major drawback of the dynamic pricing is that it is considered impractical, because the prices change on per-packet basis. For every packet that comes into the network, the provider has to decide the price of transmitting it through the network. On the other hand, the disadvantage of static pricing is that it is unfair. Specifically, users who do not fully utilize their reserved resources are charged the same price as the users who utilize all their resources. In other words, users are not discouraged from wasting all their reserved resources.

Until now, to our knowledge there is not any work which considers the programmable networks for charging. We suggest that charging on packet granularity can be better supported with 
programmable networks. We don't consider charging on coarser granularity, because bargaining long-term SLAs is an application level job, and obviously it should not be executed in the network nodes.

\subsection{How the Charging and Pricing can be Enhanced by Pro- grammable Networks}

In each border router of an ISP, which should be a programmable router, customers of this ISP (which may be third-party providers or individual users) run a code whose responsibility is to decide which classes of service to buy for each packet or for a chunk of packets. In Figure 3.1, the charging mechanism of each customer realizes his code and marks his packets with a class of service. The input parameters of this code may be the ISP's tariffs (which may be a function of the network load), the maximum cost that customer can afford, and the traffic characteristics of customer's flows. Thus, according to the current tariffs and his traffic, the customer through this code makes his decisions. Finally, the customer's code marks each packet with the class of service which he decided. In other words, customers have the flexibility to mark their packets with the class of service they want inside the home-ISP's routers.

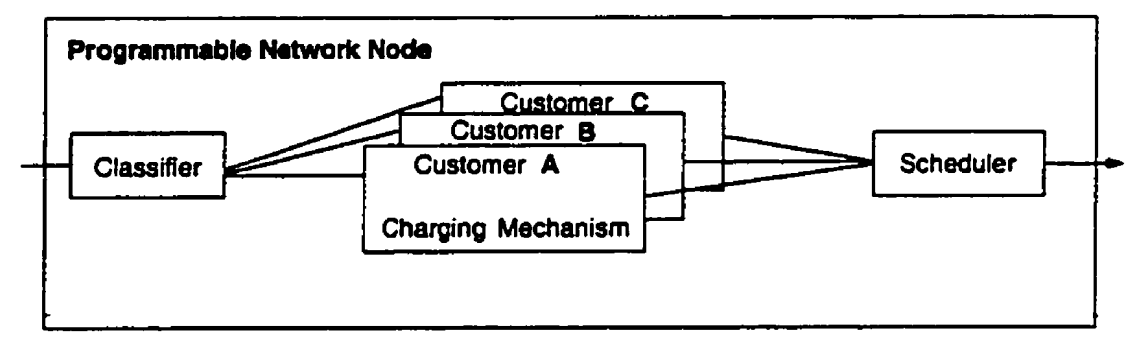

Figure 3.1: Customer's mechanisms inside the network node

The above scheme is not restricted to the DiffServ only, but it can also be extended to the auctioning mechanisms. Until now, it has been proposed that the packets should carry their bids. However, considering our mechanism the user's code can submit his bids to the auctioning task inside the network node. More specifically, instead of executing the code which computes the bids at the end-points, we propose to execute this code inside the network nodes.

Our scheme has many benefits. First, it allows customers to purchase more than one class 
of service for their traffic. In more detail, the customer's mechanism can further classify its traffic according to any field in the packet header and also according to other parameters, such as the network load. Then packets may be treated differently. Note, that traditionally the classification was based only on static parameters, such as the fields of the packet header, whereas our mechanism allows customers to classify their packets according to dynamic parameters. For example, during the peak periods, a customer buys a better class of service for the packets of a realtime application, than for the packets which belong to elastic applications. However, during the off-peak periods a customer can buy a lower quality class of service for both real-time applications and non real-time applications without experiencing any performance penalty.

Second, customers react more quickly to the network state (e.g., traffic load and tariffs), because they do not have to wait for feedback in order to make their decisions. More specifically, they show their willingness to pay inside the network node and they do not have to encode it in a field of the packet header. Likewise, providers do not have to send pricing information or any other information which is necessary for the customers for selecting classes of services for their traffic. In brief, everything operates where the parameters are being captured. This also entails to shorter delays, which in turn means that the pricing information can be updated at finer time granularity.

Third, auctioning [16] has been criticized as being impractical for many reasons. One problem is that the auctioning process takes place on a hop-by-hop basis; whereas the bids are set by the customer only at the origin of the traffic. Since the bid is set at the origin point, the customer values the service from the origin to the destination point. That means that the bid should take part in an auctioning task for getting access from the origin to destination point. However, instead of this, the bid takes part in the auctioning tasks for each hop across the path from source to the destination point. In this way, the customer should submit a bid for each hop along the path, and not a bid from the origin to the destination point. How can this be implemented? One solution is that the packet carries a distinct bid for each hop. Obviously, this is not an efficient solution. Instead, we propose to exploit the programmable networks, where each user can execute code in each network node. This code is responsible for submitting the bids to the auctioning task of this network node. What about the performance of this pricing mechanism? We do not expect any performance degradation, because the auctioning is applied only when there is congestion. Usually, congestion does not occur on each network node. This means that if there is a congestion 
in two network nodes along the path, the auctioning is executed at most twice along the path.

An issue with our auctioning mechanism is that it will be extremely difficult for the user to anticipate, even approximately, the cost of transmitting a packet end to end. That is because the bid is offered at each network node where congestion occurs and not at the origin point. However, user knows the maximum cost of transmitting a packet, which is the sum of the bids at each network node across the path. This maximum cost actually would be equal to the bid that user would offer independently of the network load, if the end to end bidding (i.e., the traditional auctioning process) was applied. Thus, the aggregate cost of sending a packet with the node by node bidding will be equal or less than the cost of transmitting a packet with end to end bidding, i.e., the user saves money with our mechanism.

Figure 3.2 depicts the smart market pricing method in traditional networks and in programmable networks. In the former case, users set their bids at the source point of their traffic, and at each congested hop, these bids either win or lose. In our example, users A and B win and the user whose bid is 15 is defeated. Thus, users $A$ and $B$ transmit their packet with cost equal to 15 (i.e., equal to the highest defeated bid). In the latter case, users set their bids in each congested hop. Note that the bids offered by the users in the first case are greater than the bids offered by the users in the second case, since in the first case the users value the service from the origin to destination, whereas in the second case users value the service for accessing only one hop. Thus, the total cost of transmitting a packet from the source to the destination equals to the sum of marginal costs in each congested link. For example the winning users $A$ and $B$ transmit their packets with cost equal to $5+2=\pi$.

In addition, the problem of sender pays or receiver pays with differentiated services (introduced by Clark and Shenker et al. $[17,16])$ can be solved. To be more specific, in DiffServ the sender marks the packets (i.e., selects the service level of his flow), and also the sender pays his local ISP for his traffic. The problem with this scenario is that there are circumstances in which the receiver is the one who should pay for service, and is the one who wants to select the service level of the traffic. How can the receiver select the class of service? One solution is to utilize the control packets (like the TCP acknowledgments packets) flowing in the reverse direction from the receiver. These packets can be enhanced to indicate the receiver's preference of class of service.

However, with our approach this problem can be solved without utilizing the control packets, and can be applied on top of the current Internet protocols. If the receiver is the natural party 

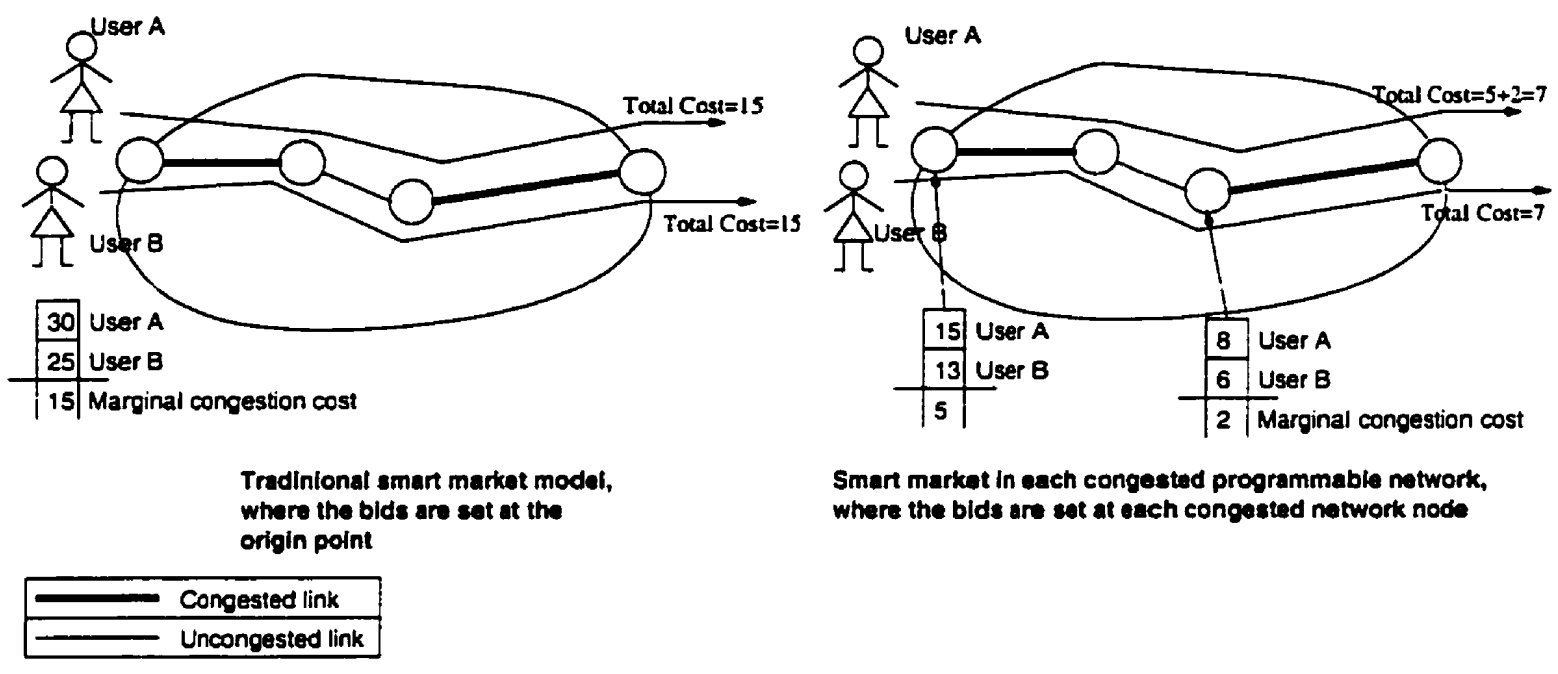

Smart market in each congested programmable network, where the bids are sat at each congasted natwork nods

Figure 3.2: Traditional smart market scheme vs. Smart-market in programmable networks

who should pay, then the receiver executes his code at the ingress network nodes ${ }^{1}$ of ISPs which should be paid by the receiver. The receiver's code is responsible for selecting the class of services for his traffic. On the other hand, if the sender is the natural party who should pay, then this is the one who executes code at the network nodes. In brief, these codes act as proxies of either the sender or the receiver, and according to each circumstance one of the proxies is selected for an ingress network node.

The above pattern can also be extended to the multicast sessions where the flow somewhere inside the Internet cloud is replicated many times. There are cases where receivers want to choose the class of service of these flows, because they are the ones who pay. A scenario can be a media server which transmits a video stream to more than one receiver. One receiver may desire a poor quality of video, whereas another receiver may desire a high quality of video. In this case, receivers should send their code to the network node where the replication takes place. Then, each receiver's code pays for the flow that it receives, independently from the others.

Finally, the user's mechanisms at end points of a connection can cooperate and allocate resources in a more effective way. For example, if a receiver's access link is bottlenecked and cannot forward the flow with the same rate as packets arrive, then the receiver's mechanism can send

\footnotetext{
'Ingress network node is a border network node of an administrative domain where the traffic flow enters into the domain. Egress network node is a border network node of an administrative domain where the traffic flow goes out of the domain.
} 
explicitly a message with the maximum capacity that can be supported to the sender's mechanism. In this way, both the sender's and receiver's mechanism allocate equal capacity without wasting resources along the path of connection. This is also useful in DiffServ, in which class of services are provided in a connection-less way. Thus, when a provider in the core of the network or the receiver's access link has insufficient capacity, either implicit or explicit congestion signals are received by the sender, although he has purchased enough capacity from his home-ISP. With the proposed scheme, along these congestion signals the receiver's mechanism can also inform the sender's mechanism of its free capacity. Then, the sender may buy less capacity, equal to the amount of free capacity at the receiver's access link.

However, the advantages of this mechanism can be negated by the overhead of CPU cycles. Another limitation is that this mechanism is not suitable for mobile networks. The reason is that, if either the sender or the receiver is a roaming user, then the path from the sender to the receiver changes dynamically, on short time scales. This induces the problem that the customer's mechanism should migrate from one network node to another network node through which the new path passes, causing the negative effect of increasing the traffic overhead.

\subsection{Example}

In this section, we describe a charging and a pricing scheme for differentiated services which was proposed for the traditional network infrastructure, i.e., was proposed to run at the end-points. This scheme is modified and it is executed in the network nodes, which are programmable nodes. Then we show how some problems are alleviated with the modified version of this scheme. Note that these problems are related to the DiffServ and not to this specific pricing model.

We assume that we have DiffServ with priorities. Specifically we have $N$ priorities, and the packets with high priorities experience less delay and are less likely to be dropped than the packets with low priorities. Since the QoSs discussed earlier do not provide strict guarantees for the network resources, we assume that customers request an elastic service.

Furthermore, we assume that the ISP does not change the prices of the priority classes on short time scales. In other words, prices are static and do not change according to the network load. However, customers are charged for each packet they transmit even if their packets are dropped. Thus, customers are encouraged to reduce their rate during periods of congestion; otherwise, they 
are charged for their dropped packets. This charging and pricing model has been proposed by Marbach [29]. One benefit of this model is that no SLAs need to be bargained. Furthermore, this algorithm has been proven to achieve equilibrium allocation.

Users execute software agents in their hosts and in every time slot these agents compute the number of packets to send and in which priority class to send, in order to maximize their net benefit. In other words. software agents compute the transmission rate of packets at each priority. The input parameters of these agents are: load of network; cost of each priority class; and the demand function of the user/application. Thus, the network nodes should send to the hosts the following information: (1) costs of their priority classes; (2) probabilities of dropping a packet in each class. Note that the costs of priority classes do not change on short time scales. Hence, it is not necessary in each time slot for the network node to send the costs. The costs can be broadcasted whenever they are changed. This scheme is depicted in Figure 3.3.

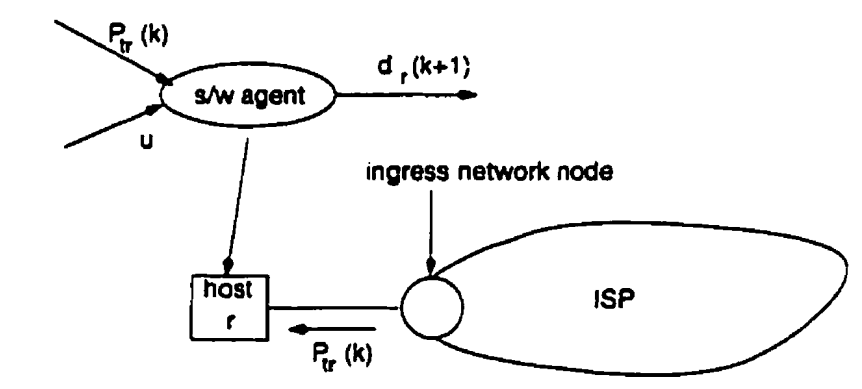

\begin{tabular}{|cl|}
\hline$P_{t}(k)$ & : probability that a packet is transmitted in the time slot $k$ \\
\hline$u$ & : costs of the priorities \\
\hline$d_{r}(k+1)$ & : transmission rate at time slot $k+1$ \\
\hline
\end{tabular}

Figure 3.3: A charging and a pricing model executed in the host at time slot $k$

We have modified the above pricing model and we run a modification of the proposed software agents in the network nodes. We assume that the applications at the host use the TCP protocol, and if the host has not received any congestion signal, then it increases the transmission rate; otherwise, it decreases the transmission rate. If the host does not decrease the transmission rate of its packets when it receives congestion signals, then it is charged even if its packets are dropped. Thus, the hosts are still encouraged to back off in the case of congestion. Similarly, the software agents in network node determine the rate of the packets in each priority class. The problem with 
executing these software agents in the border network node instead of host is that the host may have already sent more packets than the software agent has determined should be send in the next time slot. In order to overcome this problem, we propose the following solution: the redundant packets are transmitted in the same priority class but with the explicit congestion notification (ECN [38]) set. Then the receiver checks the ECN and if it is set, a message is sent to the sender to reduce his rate. When the sender does not receive such a message then he always increases the rate of the packets in each time slot. As a result, the sender adapts his rate in each priority class with the goal of maximizing his net benefit.

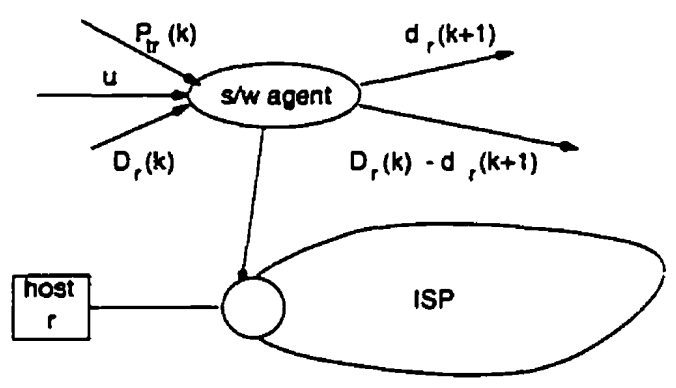

\begin{tabular}{|rl|}
\hline$P_{t}(k)$ & : probability that a packet is transmitted in the time slot $k$ \\
\hline$u$ & : costs of the priorities \\
\hline$D_{r}(k)$ & : input rates of peckets from host $r$ at time slot $k$ \\
\hline$d_{r}(k+1)$ & : rate of packets with $E C N=0$ at time slot $k+1$ \\
\hline$D_{r}(k) \cdot d_{r}(k+1)$ & : rate of packets with $E C N=1$ at time slot $k+1$ \\
\hline
\end{tabular}

Figure 3.4: A charging and a pricing model executed in the network node at time slot $k$

In this paragraph, we describe the algorithm that is executed in the network nodes by each user/application (see Figure 3.4). Let $r=1, \ldots, R$ be the users, let $k=1,2, \ldots$ be the time slots, and let $u_{i}$ represent the cost of priority $i$. In time slot $k$, user $r$ computes the number of packets which will be transmitted at each priority $i$ without having their ECN set. Let $d_{r}^{k}=\left(d_{r}^{k}(1), \ldots, d_{r}^{k}(N)\right)$ be the rate of these types of packets at the priority classes $1, \ldots, N$. The rest of the packets, the redundant packets, are transmitted with their ECN bit set. Thus, if the input rate of the packets of user $r$ is $D_{r}^{k}=\left(D_{r}^{k}(1), \ldots, D_{r}^{k}(N)\right)$, then the rate of packets having their ECN set is: $D_{r}^{k}-d_{r}^{k+1}=\left(D_{r}^{k}(1)-d_{r}^{k+l}(1), \ldots, D_{r}^{k}(N)-d_{r}^{k+1}(N)\right)$. As we have already said, the input parameters of the algorithm are: $P_{t r}\left(i, d^{k}\right)$, which represents the transmission probability for the priority class $i$ under the aggregated allocation $d^{k}$ at the time slot $k$, and $G_{r}(u)$, which is the inverse demand 
function of the user $r$. The average throughput of user $r$ is $x_{r}=\sum_{i=1}^{N} P_{t r}\left(i, d_{r}^{k}\right) d_{r}^{k}(i)$. Finally, the $\alpha_{r}>0$ is a small step size parameter, and $f_{r, i}\left(d_{r}^{k}, P_{t r}\left(i, d_{r}^{k}\right)\right)$ is a gradient direction of the net benefit with respect to $d_{r}^{k}(i)$. The step size parameter $\alpha_{r}$ is set by the user. It should be small. in order for the user's net benefit to increase. The algorithm that user $r$ executes is illustrated in Figure 3.5.

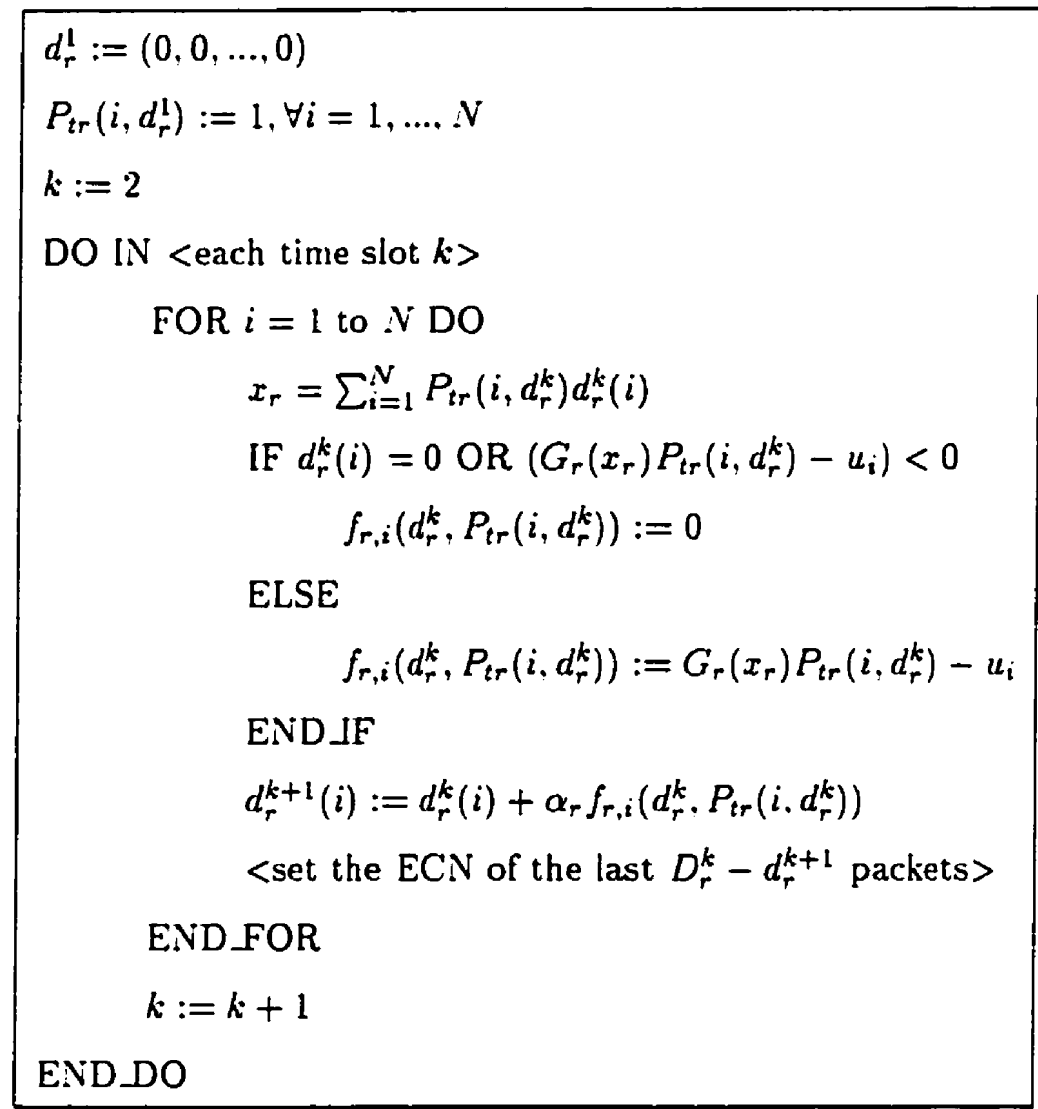

Figure 3.5: Algorithm that user $r$ executes in the network node

The first advantage of our approach is that the network state of the ingress network node is not transmitted to the hosts in each time slot, as the network node did when the software agents execute in the hosts. Thus, there is not any traffic overhead in our approach.

Another advantage is that programmable networks let the user execute his software agent in any network node. Hence, the user can update the priority of his packets according to the network load inside the network core. For example, assume that the ingress network node is underloaded. Then the user marks his packets with the lower priorities in order to increase his net benefit. 
Furthermore, assume that a network node which is crossed by his packets, is congested. Then the user can update the priority of his packets, and he can mark them with higher priorities keeping always his net benefit positive. Obviously, this is not feasible without the programmable networks. Specifically, in traditional networks, a user marks his packets according to the network load of the ingress network node, and he cannot update the priorities of his packets inside the network. If the user decides to mark his packets with higher priority because he receives congestion signals. then he decreases his net benefit, which is not desirable. However. in programmable networks, the user is able to update the priority of his packets while maximizing his net benefit and without experiencing performance degradation. 


\section{Chapter 4}

\section{A Generic Programmable Accounting Management Architecture for VPNs}

In this chapter, we describe the components and the communication interfaces of a programmable Accounting Management Architecture for VPNs. We have adopted the MIBlet architecture [37] in order to segregate the accounting information of different VPN customers. Furthermore, we provide an open accounting interface through the MIBlets. Our architecture also follows the principle of pushing the intelligence into network nodes for executing the accounting management tasks. Network nodes perform accounting tasks which traditionally were executed in centralized accounting servers.

In detail, Accounting Management Controllers (AMCs) realize the accounting tasks inside the network nodes. Some examples of these tasks are: real-time processing of metering data, monitoring high confidence traffic, compressing accounting data before transmitting them to the accounting servers, managing accounting data overloads in real-time, calculating the bids for the packets in case the auction charging model is applied, and selecting a class of service according to the current prices. There is a trade-off in our architecture: achieving more effective accounting mechanisms at the expense of more CPU cycles needed by the network processor.

Another main characteristic of our architecture is that we do not restrict providers and customers to apply their accounting tasks only inside the network elements, but we also add the concept of accounting-savvy hosts. This feature is not a new idea, but it has already been proposed by Briscoe et al. [39], who suggest that accounting tasks should be executed at the 
end-points. However, we support executing accounting, pricing and charging operations both at the end-points and at the network nodes. The choice of where these operations will finally be performed depends on many parameters which are stated in the last section of this chapter.

In the context of hierarchical VPNs, AWLCs give the freedom to VP:N customers of employing any policy and mechanism independently of their providers. Specifically, each local ISP is a VPN provider which sells its services to VPN customers. Thus, a customer is charged by every ISP providing local access to its sites. A customer has its own MIBlet which holds the accounting information of his traffic and can be accessed any time directly. Furthermore, accounting data mining can be performed by the accounting controllers of ISPs, before transmitting them to the Accounting Manager (AM).

An advantage of the MIIBlet controllers and the AMCs is that the VPN customer can retail the network resources to its users, i.e., the VPN customer can act as a third-party provider. This is feasible, because the MIBlet controllers let the third-party provider maintain its own accounting group independently of the accounting group of VPN provider. Moreover, the third-party provider can execute any charging, pricing and accounting mechanism, without being restricted by his provider.

In this scenario, the users are charged by the third-party provider, and they do not need to have specific knowledge of the charging and pricing schemes adopted by the local ISPs of their end-points, which most probably will not be the same. (Each ISP will adopt and will execute its own techniques.) Instead, users have a general view of the Internet infrastructure, and they exchange pricing information, such as bargaining SLAs, only with the third-party provider. On the contrary, the third-party provider has to deal with the heterogeneous charging schemes of ISPs, and has to map these schemes into a uniform accounting system for its users. The AMCs realize this mapping. For example, if a user asks for premium service from his third-party provider, then the provider maps this premium service to the similar service supported by the local ISPs.

The overall accounting management architecture in a single VPN is depicted in Figure 4.1. There are three major components which are involved in our architecture: Accounting Management Controllers (AMCs), MrBlet Controllers, and Accounting Manager (AM). The first two components are in the resource management layer and the last one is in the network management layer. Figure 4.1 illustrates AMCs being executed at the hosts of both a roaming user and a dial-up user, and at the border routers of local-ISPs of the two sites ('Site 1' and 'Site 2'). 


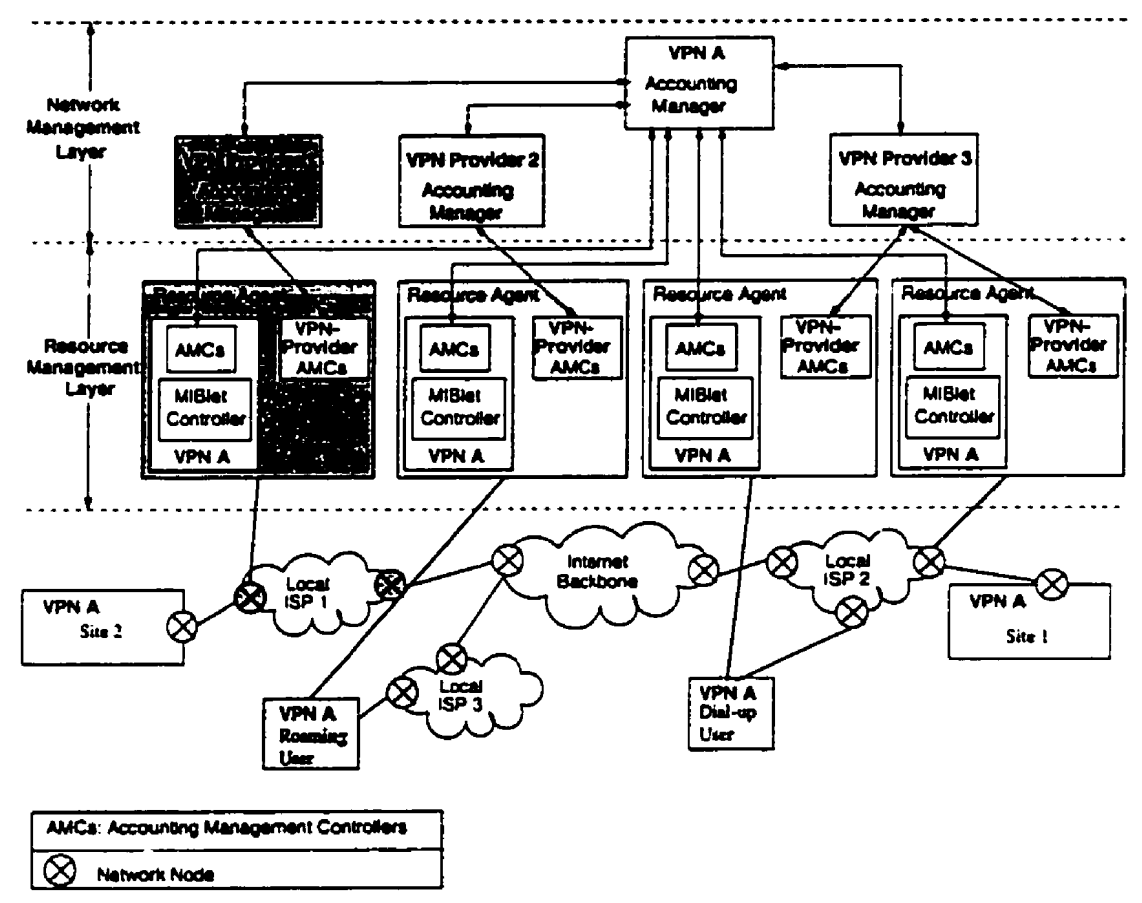

Figure 4.1: Overall Accounting Management architecture in a customer VPN. called VPN A

The VPN Provider executes its AMCs in the Resource Agent of its network nodes. In addition, its customers, also, execute their AMCs. The AMCs are installed in specific network nodes and/or in its end-points. These network nodes can be either at the borders of local ISPs of the VPN or at any network node in the Internet core. This depends on how the VPN service is provided by the ISPs and also on the QoSs. If, for example, DiffServ are supported, then accounting takes place only at the border network routers of local ISPs.

In each VPN provider, there is one Accounting Manager. The Accounting Manager is responsible for collecting resource consumption data (which may be already processed by the AMC) which are then used by accounting applications such as capacity and trend analysis, cost allocation, auditing and billing. The AM charges its customers, who consume its networking resources. It can also provide some accounting services to its customers. For example, it can provide a service for real-time charging where the customer can see the current status of his invoice. Another example is the pre-paid debit card, where the customer pays in advance the provider and then the provider executes the appropriate accounting controllers in specific routers. Then, the controllers decrease the customer's credits whenever he uses the resources. 


\subsection{Communication Interfaces for Accounting}

There are mainly three communication interfaces: (1) communication between the Accounting Management Controllers/MIBlet Controllers and the Accounting Manager, (2) communication between the Accounting Manager of the VPN provider and the Accounting Manager of the customer, and (3) communication among the Accounting Management Controllers. In the following paragraphs, we describe only the type of messages that are exchanged among the accounting components, and we do not give a thorough description.

\subsubsection{Communication Interface between the AMC/MIBlet Controller and the} AM

In a network node, both the provider and the customer execute their AMCs. The AM of a provider communicates with its AMCs, and respectively the AM of a customer communicates with its AMCs. Furthermore, the customer executes its own MIBlet Controller.

There are two types of information exchanged between them: control information and accounting information. Control information is sent to the controllers, in the case that they have to be configured. For example, if the customer is a third-party provider and he has to track the traffic of a new user, then the AM of the customer has to send a control message to the MIBlet controller in order to maintain the resources consumed by the new user. Another example is when charging and pricing take place in the network node, then the customer configures his charging controllers with his utility functions.

The Accounting Controllers are also responsible for sending the accounting records to the AM. These records can be transmitted by a standard protocol, such as DIAMETER or COPS. We have already mentioned that IETF tries to provide a set of tools for retrieving the accounting data.

\subsubsection{Communication Interface between the AM of the VPN-provider and the AM of the VPN-customer}

In the Network Management Layer, the AMs of the provider and the customer exchange high-level information. More specifically, first the customer informs the provider of the network topology of the VPN it wants to set up. This includes IP addresses of the LANs of its sites, and roaming users. Moreover, the VPN provider allows the customer to add or delete end-points in the future. 
Furthermore, they exchange pricing information which depends on the QoSs the VPN provider supports. For example, they negotiate SLAs automatically, which carry service provisioning, routing and pricing information [40]. This is suitable for the DiffServ.

Finally, the customer may request to access its account status on short time scales. Thus, the provider should be able to collect the accounting data, process them and send the current status of the customer in real time. Each month or at predetermined times, the provider issues invoices and sends them to the customers.

\subsubsection{Communication Interface between the AMCs of the VPN-provider and the AMCs of the VPN-customer}

When charging operations take place inside the network nodes, then A.MCs of the provider should inform the AMCs of the customers of the tariffs in each interval. Another example is when the custumers have to bid for their packets. In this case, the customer has to inform the provider of his offer.

This interaction takes place through the MIBlet controllers. To be more specific, a provider allows read-only access to the entries of MIB that the customer should know, such as tariffs. Furthermore, the provider lets the customers have write access to specific entries of MIB. For example, these entries can consist of the bids of the customers.

\subsection{Accounting Components in the Network Node}

There are three accounting components (Figure 4.2) in the resource management layer: accounting management controllers (AMCs), customer's MIBlet Controllers, and an Accounting Group Controller of the provider. Each customer executes its own accounting policies through the installed accounting management controllers. The AMC gets the accounting data from the accounting group in the MIBlet.

\subsubsection{Accounting Group Controller and MIBlet Controller}

The Accounting Group Controller acts as a meter. Furthermore, it maintains its own MIB with an Accounting Group, or it updates the accounting group of the standard MIB. Finally, the Accounting Group Controller keeps statistics for the customer's traffic, which are used by the 


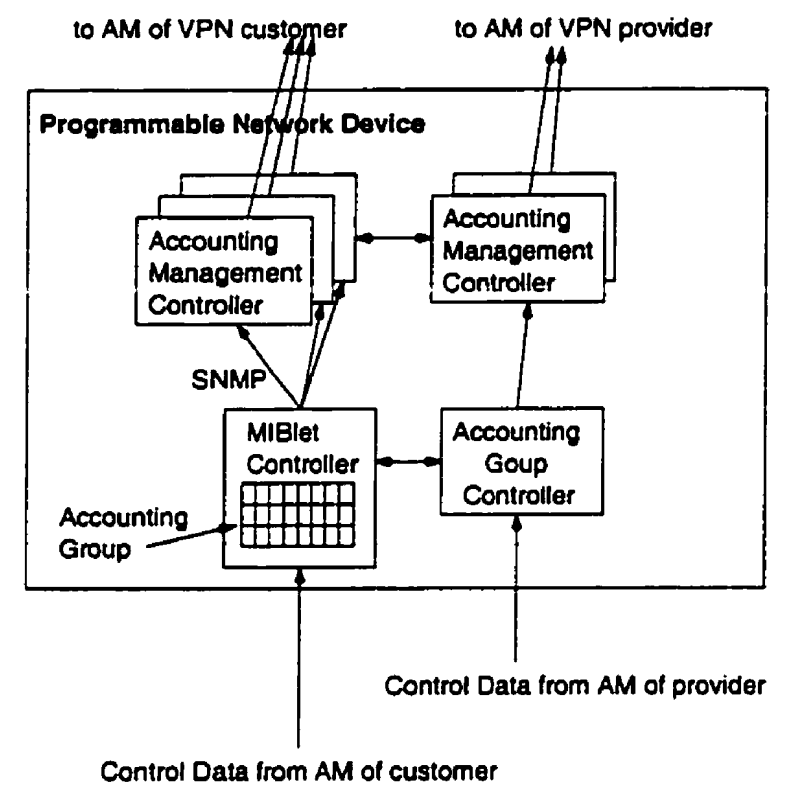

Figure 4.2: Accounting Components in a network node

charging and pricing tasks. Each network node has an Accounting Group controller for either the input or output physical links. (Otherwise duplicate accounting data would exist per physical link.) This controller is configured to identify sessions of distinct VPNs.

The AM configures the Accounting Group Controller to monitor the traffic according to any fields of the headers of the packets. For example, it can measure the packets originated at and destined to specific IP addresses. For each customer, there is a row in the Accounting Group which tracks the network resources consumed by this customer.

The MIBlet Controller is an Accounting Group Controller for the customer. The main difference is that the customer's controller can monitor only its traffic, whereas the provider's controller can monitor any traffic that crosses this network node.

\subsubsection{Accounting Management Controllers}

We can classify the Accounting Management Controllers according to their tasks. Thus, we have:

- Charging and Pricing Controllers: These controllers are executed when charging granularity is in terms of packets. They realize the mechanism we have proposed in Chapter 3. Some of their parameters can be received by the AM (such as the utility functions) and 
some other parameters may be maintained in the MIBlet controllers (such as the current tariffs of the classes of services).

- Accounting Controllers: These controllers are mainly responsible for the processing of accounting data. They can communicate with the AMI or not. For example, the controller which is responsible for decreasing the credits of the customers has no reason to communicate with the AM. On the other hand, if the accounting controller compresses the accounting data, then it sends them to the AM.

\subsection{Accounting Components in the Host}

The Accounting Manager has the option to send specific AMCs to the edges (i.e., hosts) of a VPN in addition to the network nodes. Thus, AMCs exist not only at the core of the network, but also at the edges. The advantages of host A.MCs are explained at a separate section (Section 4.5). Similarly to the network node, there is also an Accounting Group Controller which measures the traffic produced or received by this host.

\subsection{Accounting Manager}

There is an Accounting Manager (Figure 4.3) for each VPN customer which executes at the Network Management Layer. The components of an Accounting Manager will be automatically launched upon the setup of the first customer. Later, these components will be configured to charge other customers. The fundamental building blocks of the Accounting Manager are described briefly in the following sections:

\subsubsection{Aggregator}

The aggregator is responsible for gathering the accounting data received from the network nodes and from the end-points. In addition, it correlates and stores these data in an accounting database which is read by the accounting applications. 


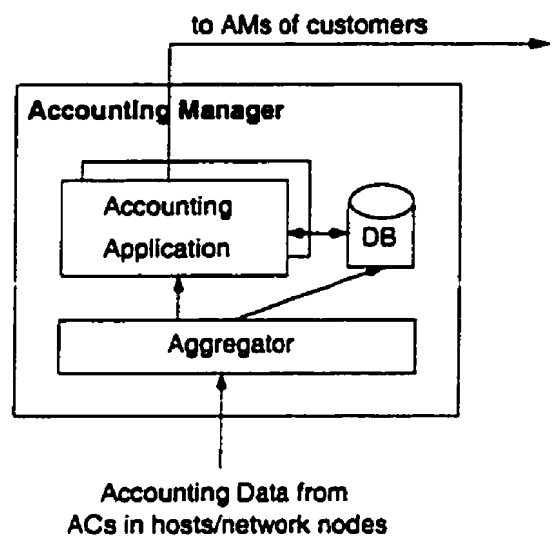

Figure 4.3: Architecture of Accounting Manager

\subsubsection{Accounting Application}

The accounting applications are: accounting planning, auditing, charging, and billing. Some applications communicate with customers. For example a billing application sends invoices to customers.

\subsection{Discussion of Having AMCs Both at Hosts and Network Nodes}

In this section, the advantages of executing AMCs inside the network and at the edges are described. The obvious advantage of having both routers and hosts programmable is the immediate installation of new protocols and applications.

One benefit of host Accounting Group Controllers is that they can alleviate the metering inside the network. Thus, if metering is executed at the end-points, then the network nodes do not have to perform any metering because the AM of provider retrieves the accounting data from the end-points. Having meters at edges, the issue of security is raised. Users can replace the legal meters with their own meters, and thus they can deceive accounting managers. This can be avoided through the authentication of accounting data produced by the host AMCs. Another way of overcoming this problem is to have the network nodes meter samples of traffic crossing through. this network node. Then the manager can decide whether the metering data transmitted by hosts are valid or not, by examining the difference. 
Another gain of host AMCs is that, if the routers are not programmable, then they cannot meter packets according to the fields of network layer 4 (i.e., transport layer) or higher. However, hosts can perform metering in terms of any field of their packets.

Moreover, when per packet charging is applied, our decision for charging in the hosts or in the network nodes depends on many parameters. First, it is better to execute the charging mechanism in the host when the host executes applications that are adaptive to the traffic load or to the tariffs. The drawback of executing the charging mechanism in the host is that it has to await feedback signals which carry information about network load or carry the tariffs of the network resources. However, the charging mechanisms in the network node do not have to await any signal, and there is no delay for setting the prices from the provider standpoint, and no delay for selecting a class of service from the customer standpoint.

The comparison of AMCs in hosts and routers is described briefly in Table 4.1.

\begin{tabular}{l|l|l} 
& Charging and Pricing AMCs & Accounting AMCs \\
\hline $\begin{array}{l}\text { Programmable } \\
\text { Routers }\end{array}$ & $\begin{array}{l}\text { (1) no-delay for setting the prices } \\
\text { (2) no-delay for selecting a class of } \\
\text { service }\end{array}$ & $\begin{array}{c}\text { (1) fine-grained metering at the } \\
\text { expense of requiring more } \\
\text { CPU cycles }\end{array}$ \\
\hline $\begin{array}{l}\text { Programmable } \\
\text { End-points }\end{array}$ & $\begin{array}{c}\text { (1) suitable for adaptive } \\
\text { applications network load } \\
\text { and tariffs }\end{array}$ & $\begin{array}{l}\text { (1) fine-grained metering } \\
\text { (2) requires security services }\end{array}$
\end{tabular}

Table 4.1: Host AMCs vs. Router AMCs 


\section{Chapter 5}

\section{Case Study}

In this section, we describe an example of charging, pricing, accounting and billing VPNs. ISP providers are assumed to support VPN services. Thus, each site of the customer requests a QoSenabled VPN service from its local ISP. We have adopted the edge-pricing model [16], which means that VPN-customers pay only the local ISPs of their sites, and in turn the local ISPs pay their neighbour ISPs. Figure 5.1 illustrates our scenario, in which a VPN Customer has two Sites, named $\mathrm{A}$ and $\mathrm{B}$, which are attached to two different local ISPs. Thus, a customer requests a VPN service from these two local ISPs, and is charged by these two ISPs.

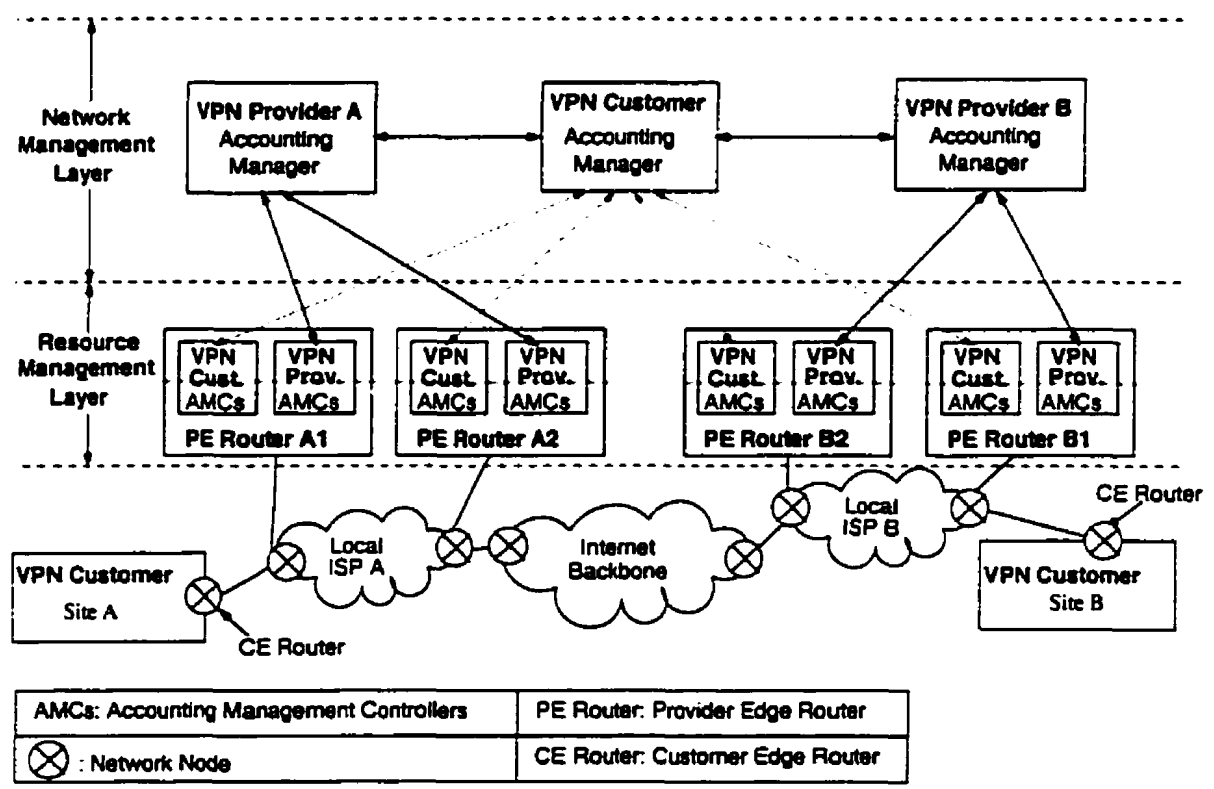

Figure 5.1: A VPN Customer with two sites: Site A and Site B; customer requests VPN Service from its two Local VPN Providers: Provider A and Provider B 
We assume that ISPs support Multiprotocol Label Switching (MPLS [41]) VPNs. There are many approaches for supporting VPNs that use MPLS. We adopt the standard BGP/MPLS VPN [13]. This type of VPN solution is based on the peer model, where the service providers act as "peers" of the customer networks, and the customer edge routers (CE) communicate with the provider edge routers (PE) directly. In the next paragraphs, we outline this specific VPN solution.

As the name suggests, the protocol that is used for distributing the routing information in an Autonomous Area is the interdomain routing protocol BGP, and the technique that is used for constraining the routing distribution is the BGP community. If in the same PE there are more than one CEs from different VPNs. then each CE of a VPN is connected to different interface in the PE. The PE identifies the VPN of a packet from the interface through which it arrives. Moreover, a PE maintains multiple forwarding tables, one per VPN. Thus, when the VPN of a packet is identified, then the PE uses the forwarding table associated with that VP. A. CE propagates its route to the PE using any routing protocol, such as RIP, OSPF or BGP. Another issue is that two sites from different VPNs can have the same IP address. Thus, the provider's BGP cannot establish a route, if two edges have the same IP, because it assumes the IP addresses are unique. A solution to this problem is the use of VPN-IPs which are configured by the VPN's provider. Specifically, the VPN's provider concatenates the IP with a Route Distinguisher and constructs the VPN-IP address, which is distinct for each VPN. However, the IP header of packet from a VPN carries the IP address and not the VPN-IP address, which is only carried in the routing protocols. In brief, BGP is used to construct the routes using the VPN-IP addresses.

The MPLS is used for forwarding the IP packets along these VPN-IP routes constructed by the BGP. Thus, the PE router converts unlabeled packets into labeled ones and vice versa. More specifically, it binds a VPN-IP route with a Label Switched Path (LSP) and then forwards the IP packets along this route using the MPLS as the forwarding mechanism. For scalability reasons, a hierarchical routing is employed. By using this technique, the routers along the path do not maintain any routing information for the VPNs. Routers only have the routing knowledge to forward the packets to an egress PE. On the other hand, the egress and ingress PEs have the routing information for forwarding the packets to the CE of the VPN to which they belong. This is accomplished by using two labels in the packet's header: the first-level label is used for forwarding the packet from the ingress $\mathrm{PE}$ to the egress $\mathrm{PE}$ and the second-level label is used to forward the packet from the egress PE to the VPN. The first-level label is distributed via Label 
Distribution Protocol (LDP) or, if a service provider wants to use traffic engineering, via RSVP or Constraint-based Routing LDP (CR-LDP). The second-level label is distributed by BGP, as described in the previous paragraph.

The QoS that are delivered by the local ISPs are differentiated services. More specifically, there are two services: one for real-time traffic and one for non real-time traffic. The scheduling discipline is capacity based, and it is assumed that the buffers of real-time service are larger than the buffers of non-real time service. Moreover, in this type of service, the maximum queuing delay is loosely guaranteed (i.e., the average level of performance is ensured). The Service Level Agreements (SLAs) that are exchanged among the customers and providers contain the following traffic characteristics $(x)$ : peak rate denoted by $h$, and a token bucket $(\rho, \beta)$, where $\rho$ is the token rate and $\beta$ is the bucket depth.

The solution that we have adopted in order to support DiffServ over MPLS is to mark the Exp field (experimental field). Since ISPs support only two classes of services, we need just one bit in the Exp field ${ }^{1}$ for marking the class in a packet. Thus, the labels show where to forward the packet and the Exp bits show the class of service of the packet. We should emphasize that a Label Switch Path (LSP) is shared by many origin-destination pairs, whereas the paths in AT.M and Frame Relay are used by only one origin-destination pair. This is a basic principle of MPLS, which yields scalability advantages.

Finally, both local ISPs measure the resource usage considering the notion of effective bandwidth. The power of effective bandwidth is that it permits the resource usage aggregations of a broad range of source types, thereby encoding a multiple-dimension service quality guarantee into a one-dimensional bandwidth reservation without efficiency loss $[42,43]$. Thus, the constraint on link utilization can be expressed as a linear and one-dimensional function: $\sum_{i} \alpha\left(x_{i}\right) \leq K$, where $\alpha\left(x_{i}\right)$ is the effective bandwidth, (i.e., a measure of the resource usage) consumed by the contract $x_{i}$, and $K$ is the effective capacity of the link (which depends on the capacity, buffer, and QoS). In our case, where the traffic profiles are expressed by the triple $\{h,(\rho, \beta)\}$ the effective bandwidt $h^{2}$ depends on the traffic contract parameters and on simple measurements, such as the mean rate denoted by $m$. Thus, resource usage we consider is:

$$
\alpha(x, m)=\frac{1}{s t} \log \left[1+\frac{m}{H(t)}\left(e^{s t H(t)}-1\right)\right]
$$

\footnotetext{
'The length of the Exp field is 3 bits.

${ }^{2}$ Actually we use an approximation of actual effective bandwidth, because the effective bandwidth expressions are in general complex functions requiring knowledge of traffic statistics, which are unknown in most cases.
} 
where $H(t):=\min \{h, \rho+\beta / t\}$ (referred as effective peak) and denotes the maximum amount of traffic that can be sent in time interval $t$. The parameters $s, t$ (space and time parameters) depend on the link resources (capacity and buffer) and the characteristics of the multiplexed traffic.

We will not give more details about the theory of the effective band width, because it is beyond the scope of this thesis. We just want to emphasize that problems in admission control, network resource dimensioning, and pricing can be simplified with the use of an accurate and unifying definition of resource usage, i.e., with the use of effective bandwidth.

\subsection{Charging and Pricing Techniques}

Each ISP is free to employ any charging and pricing model. Lintil now. VPNs have been supported by leased lines, which deliver guarantees of services; hence VPNs will continue to require multiple levels of QoSs from their provider. This entails that pricing techniques whose goal is to avoid congestion without providing multiple QoSs are not suitable for our scenario. Briefly, the goal of the charging and pricing models that we have adopted is to encourage customers to request the appropriate SLAs for their streams. Customers cannot "cheat" their providers; otherwise they will be charged more money. In this section, we describe the charging and pricing models that the two local ISPs apply and also the code executed by the pricing controllers of VPN customer and providers.

In the local ISP B, we assume that the pricing model proposed by Courcoubetis and Siris [33] is applied. In the next paragraphs, we outline this model. The prices of SLAs are set by the providers and are modified on long time scales. The provider posts the value of time parameter $t$ and a family of pricing curves $f_{H}(m)$ parameterized by $H$. The reason that the provider also posts $t$ is that this parameter helps the customers to select the optimal token bucket parameters. (This is discussed in the next paragraph.) The pricing curves are given by $f_{H}(m)=p a(x, m)$, where $p$ is the price per unit of effective bandwidth. Thus, the pricing curve is increasing in $H$ (i.e., the effective peak) and increasing and concave in $m$ (i.e., the mean rate). The value of $m$ is measured at the ingress network node. Thus, if a customer selects a contract $x=\{h,(\rho, \beta)\}$, then his charge will be $f_{H}(V / T) T$, where $V$ is the volume transferred in that period. As we have already mentioned the prices are adjusted in every average time periods of the duration of SLAs. Specifically, a provider computes $\sum_{i} \alpha_{i}$ and if it smaller than the expected capacity $k$, then the prices are decreased; otherwise, the prices are increased. Figure $\mathbf{5 . 2}$ shows the provider functions. 
Note that these functions are performed at the Network Management Layer.

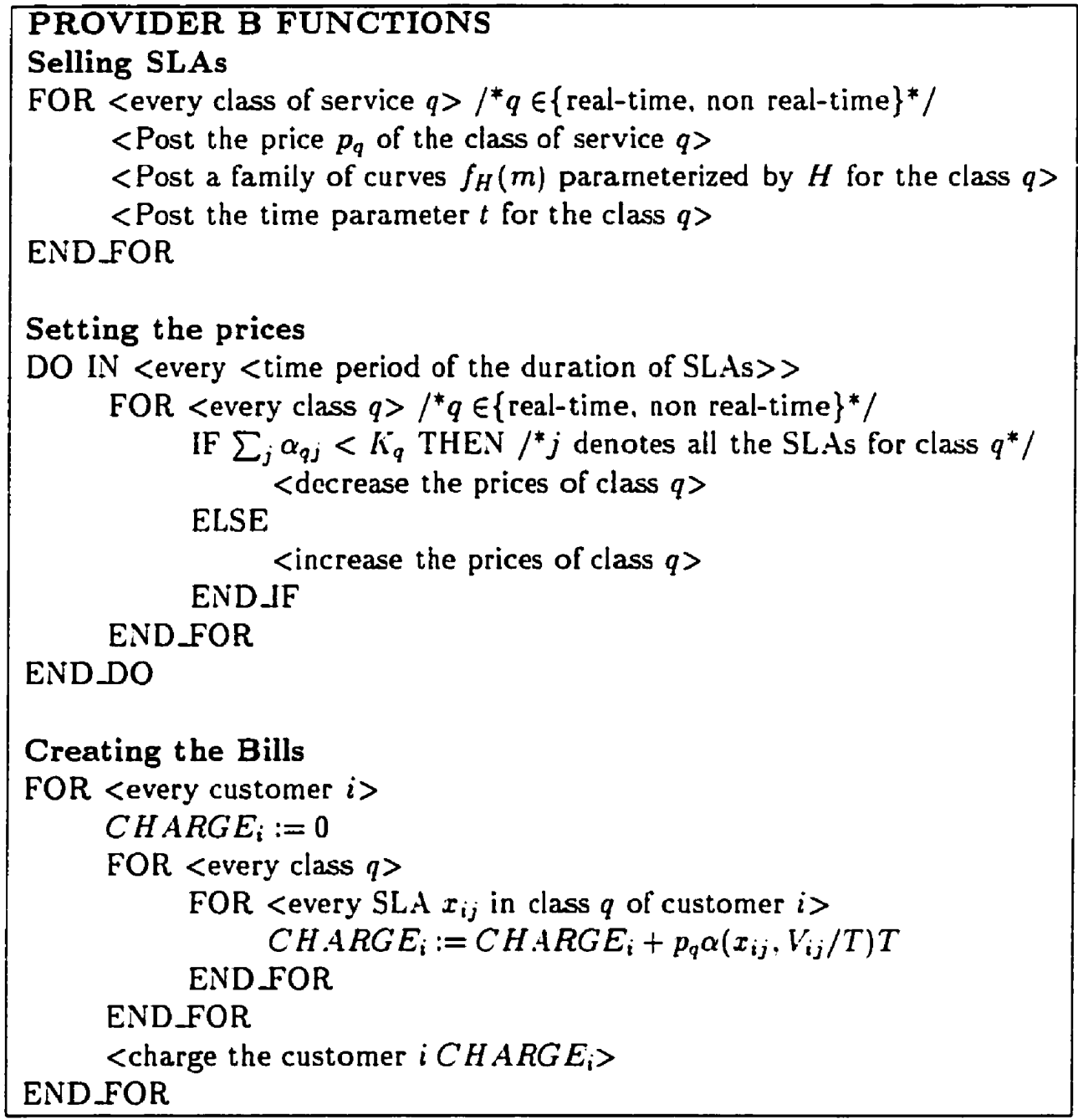

Figure 5.2: Provider B functions, which are performed at Network Nanagement Layer

The customer wishes to select a traffic contract $x$ that minimizes his cost. The traffic parameters depend on the type of the traffic. Thus, a user can have a database of pairs: applications and their traffic parameters. This database can be created based on past measurements collected by the customer. Briefly, peak rate depends on the amount of shaping, and a larger amount of shaping means a larger delay before transmitting the data to the network. Furthermore, a user can select the token parameters $(\rho, \beta)$ based on the percentage of non-conforming traffic. Recall that the effective bandwidth is increasing in the effective peak $H$, and that the customer's charges are increasing in the effective bandwidth. Thus, the customer should select such token parameters, 
so that the $H=\min \{h, \rho+\beta / t\}$ is minimized. The pair $(\rho, \beta)$ that minimizes $H$ is given by the tangent to the indifference curve ${ }^{3} G$ with slope $-t$. The time parameter $t$ is known, because the provider has already posted its value. Figure $\mathbf{5 . 3}$ shows the customer function.

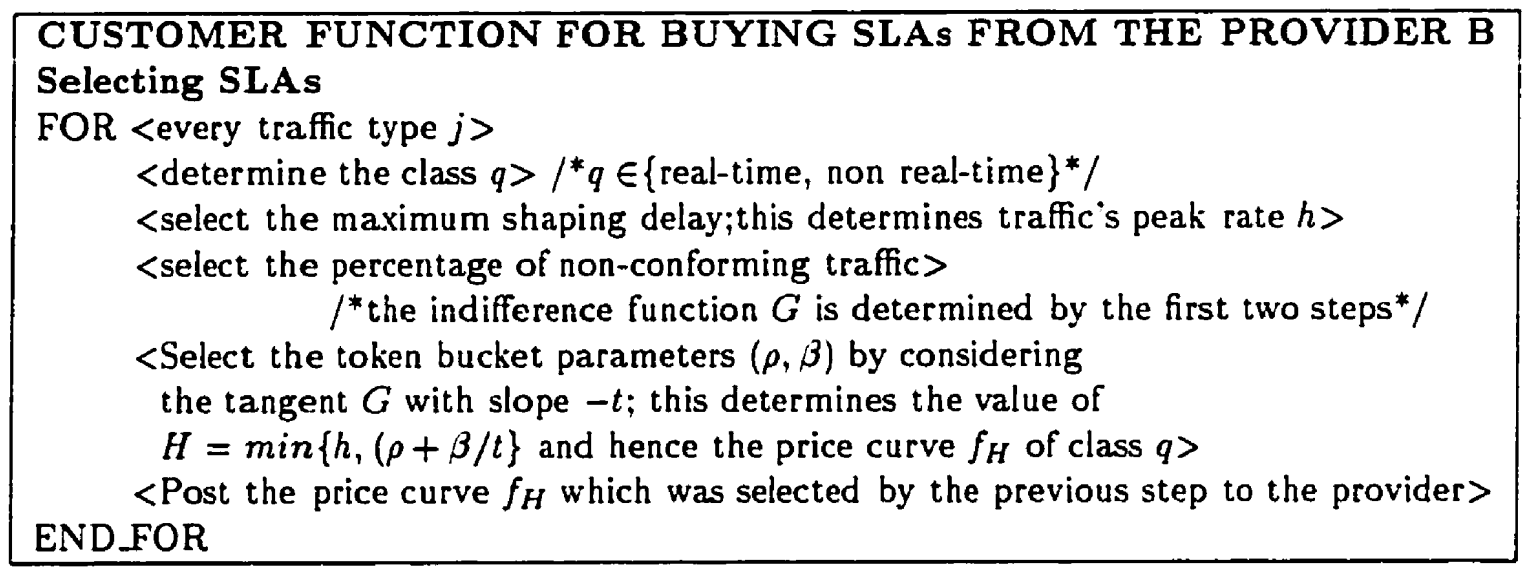

Figure 5.3: Customer function for buying SLAs from the provider B, which are performed at Network Management Layer

On the other hand, the local ISP A employs a real-time pricing model. A provider auctions each unit of its effective bandwidth in each of its classes of services (real-time and non real-time services). Auctioning takes place in all of the ingress nodes of ISP. If an ingress node is not congested, then there is no reason for the provider to auction its resources. On the other hand, provided the ingress node is congested, then at specific time intervals, called auctioning intervals, the effective bandwidth of both classes is auctioned. Finally, a provider charges its customers a fixed amount of money for the network infrastructure (that includes cost for accessing the links and network nodes and the salary for the support staff). The mechanisms of the real-time pricing model are implemented in the Resource Management Layer.

In every auctioning interval, each customer tries to reserve effective bandwidth for each service class and for each of its types of traffic. The duration of this interval is $T$. Thus, every $t$ time units, customer $i$ sends his bid for each of its types of traffic, denoted by $j$. The traffic characteristics of type $j$ are denoted by $x_{i j}=\{h,(\rho, \beta)\}$. Let $b_{i j}$ be the bid sent by customer $i$ for his traffic type $j$, and let $\alpha\left(x_{i j}, V_{i j} / T\right)$ be the amount of effective bandwidth customer $i$ wants to consume. The $b_{i j}$ is given by the utility function, which is expressed as a function of $h$ and $(\rho, \beta)$. The effective

\footnotetext{
${ }^{3}$ The indifference curve is formed by the pairs $(\rho, \beta)$ for which all of the user's traffic is conforming to specific traffic parameters
} 
bandwidth $\alpha\left(x_{i j}, V_{i j} / T\right)$ is calculated by Equation 5.1, and the space and traffic parameters $(s, t)$ are different for each service class. The algorithm of a customer's functions is shown in Figure $\mathbf{5 . 4}$

CUSTOMER's $i$ FUNCTION FOR BUYING RESOURCES
FROM THE PROVIDER A
Post the bids to the provider
FOR <every traffic type $j>$
<determine the class $q>/^{*} q \in\{\text { real-time, non real-time }\}^{*} /$
<select the $b_{i j}$ by considering the utility function>
<post the triple $\left\{b_{i j}, \alpha\left(x_{i j}, V_{i j} / T\right), q\right\}$ to the provider>
END_FOR $/^{*}$ the $\alpha()$ functions are different for each service class $q^{*} /$

Figure 5.4: Customer functions for buying resources from the provider $A$, which are performed at Resource Management Layer

When a provider receives all bids from all the customers, it normalizes them, i.e., it computes the price per unit of effective bandwidth. Let $v_{i j}$ be the the price per unit of effective bandwidth, submitted by customer $i$ for his traffic type $j$. Then $v_{i j}=\alpha\left(x_{i j}, V_{i j} / T^{\prime}\right) / b_{i j}$. The provider sorts all the $v_{i j}$ for each $i$ and $j$. Then, the provider selects the highest $v_{i j}$, subject to $\sum_{i, j} \alpha\left(x_{i j}, V_{i j} / T\right) \leq$ $K$, where $K$ is the effective bandwidth of the output link. Let $v^{\prime \prime}$ denotes the highest bid among the bids for the traffic which cannot be transmitted at the next time interval (i.e., among the bids of the defeated traffic streams). The $v^{\prime \prime}$ is called the marginal cost, or market-clearing price. Then, the price per unit of effective bandwidth in the next time interval is equal to $v^{\prime \prime}$. Thus. all the winning customers will be charged $v^{\prime \prime} \alpha\left(x_{i j}, V_{i j} / T\right) T$ and they will have surplus equal to $\left(b_{i j} \alpha\left(x_{i j}, V_{i j} / T\right)-v^{\prime \prime} \alpha\left(x_{i j}, V_{i j} / T\right)\right) T$ for their winning traffic types $j$. Note that the traffic that cannot be transmitted will be buffered. The algorithm of the provider function is shown in the Figure 5.5.

\subsection{Metering, Accounting Protocol and Billing}

In this section, our architecture is described. First, we define the functional blocks of the architecture, and then we define the communication interfaces among them.

\subsubsection{Functional Blocks of Accounting Management}

In this section, we define the functional components in the Resource and Network Management Layer. Specifically, we describe where the above algorithms are executed in our architecture. 


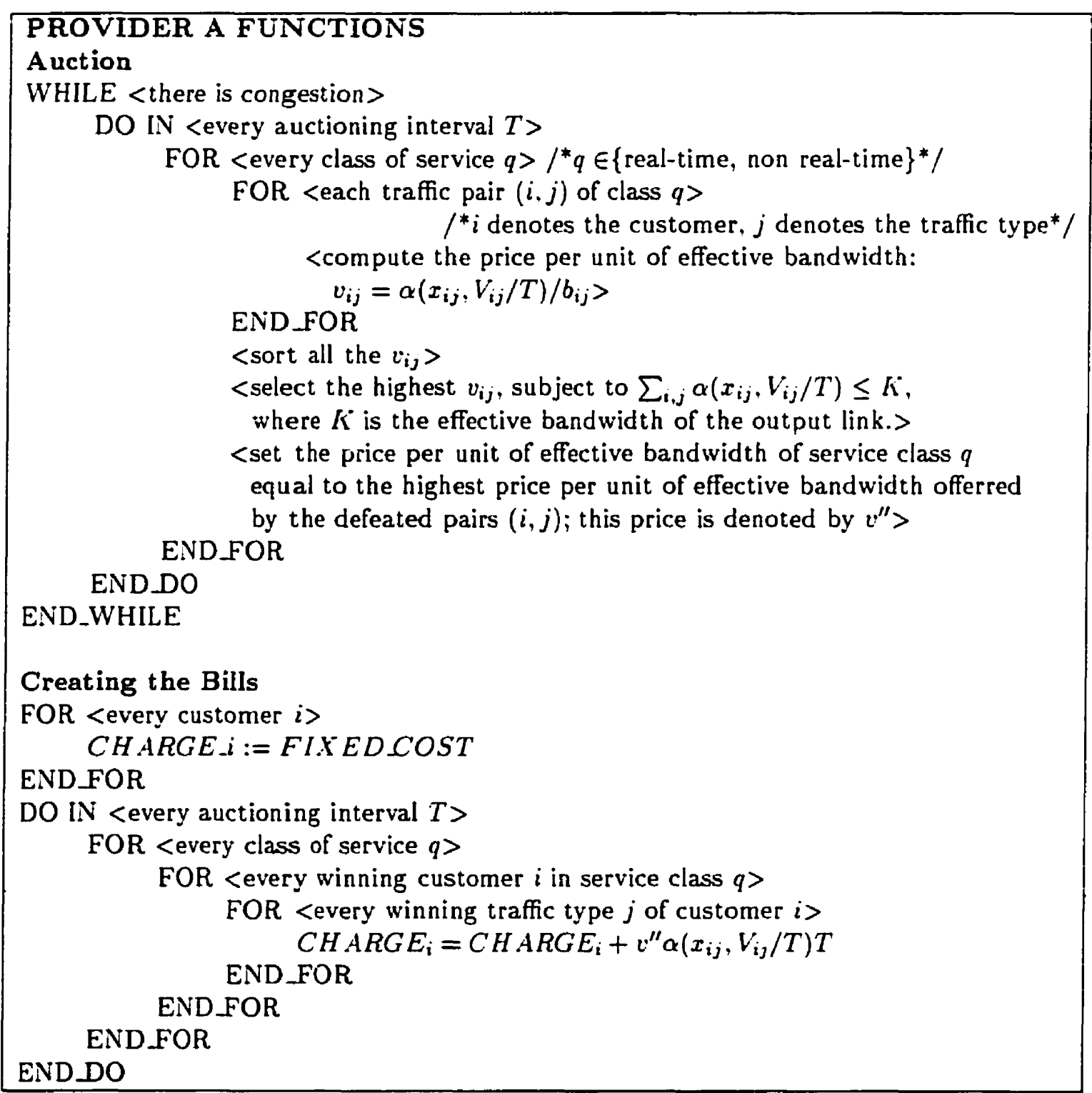

Figure 5.5: Provider A functions, which are performed at Resource Management Layer 
Furthermore, all the other components, which actually do not participate in the accounting management, are described briefly. More information for the other components is given by $\mathrm{Ng}$ et al. [37].

The components in the Network Management Layer are (see Figure 5.7):

- Accounting Manager (AM): the AM performs the tasks of accounting in the Network Management Layer. The VPN Provider initializes one AM per customer. This gives the flexibility to the provider of applying different policies between the customers. For example. one customer could request real-time accounting, whereas another customer could request a monthly accounting. Each $A M$ in the provider interacts with the corresponding $A . M$ in the customer's side. To put it differently, the AM in provider acts as a server and the AM in the customer acts as a client. The accounting tasks in each AM are (see Figure 5.6): Billing, Pricing, maintaining an accounting database, and aggregating.

To be more specific, the Aggregator collects the accounting data of a specific customer from many ingress routers. Note that there are at least two ingress nodes for each customer. For example, in Figure 5.1 the two ingress routers in Provider $\mathrm{A}$ are: "Al" for the traffic originating at the "Site $A$ " of customer; and "A2" for the traffic originating at the "Site $B$ " of customer. Accounting data are aggregated at the Network Management Layer and are stored in the accounting database.

The accounting records in the accounting database can be very detailed (e.g.. the resources consumed by each application of the customer) or can be coarse (e.g., the resources consumed by each site of the customer). It is similar to the accounting summaries we get from the telephone companies. The basic fields of an accounting record are: (1) the effective bandwidth that was consumed, (2) the period that was consumed, (3) in which network node it was consumed, (4) the price per unit of effective bandwidth in that period.

In Provider B, where the charging and pricing is performed in the Network Management Layer, there is also the Pricing task. This task executes the algorithm "Setting the prices" in Figure 5.2. The pricing and charging in Provider $\mathrm{A}$ is performed in the Resource Management Layer, and it will be explained later.

Finally, the task which interacts with the customer is the Billing. In Provider B, when the VPN is created, the algorithm "Selling SLAs" in Figure 5.2 is performed. Whenever the 
Billing sends the accounting information of a customer, the algorithm "Creating the Bills" in Figure 5.5 and in Figure 5.2 is executed in Provider A and B respectively. Note that these algorithms compute the total expenses of customers. However, they can be easily modified to issue a bill with finer granularity, i.e., a detailed bill.

On the customer's side, the Billing process is just a client application, which displays his accounting summary. Specifically, interaction between the customer and provider is done through the usage of web browsers and applets. The customer downloads an applet from the provider that communicates with the web server of provider, and this applet can display the current status of a customer's accounting. In this way, the interaction between the customer and provider is automated
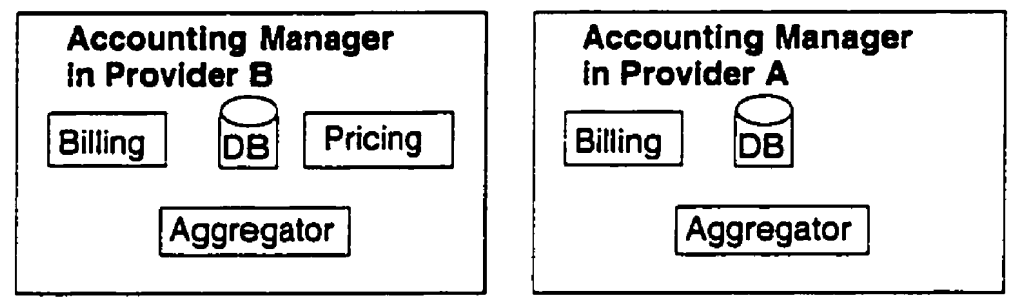

Figure 5.6: Functional Blocks in the Accounting Manager of Provider A and B. In Provider A there is not any "Pricing" component, since the charging and pricing is performed in the Resource Management Layer

- Accounting Classifier: This component receives the accounting records from the ingress nodes and classifies them according to the customer to whom they belong. In our example, customers are distinguished by the first-and second-level labels. Then the classifier forwards the accounting records to the appropriate A.M.

- AMCs Manager: this component maintains the database with the accounting controllers. Furthermore, the AMCs Manager is responsible for injecting the controllers to the network nodes.

- Resource Manager: this component acts as a bandwidth broker. It is used by the customer in order to coordinate the resource allocation among his local ISPs. More specifically, it maintains the resources that are allocated in each ingress network node of "Site $A$ " and "Site B" for each traffic type. Thus, if there is congestion in one network node and the accounting controller of the customer cannot allocate enough resources, then the controller 
sends a message to the Resource Manager in order to inform it of the maximum resources it can allocate. Then, the Resource Manager informs all the other controllers in ingress network nodes for reducing their allocated resources. This has the advantage of allocating the resources more efficiently. Note that the number of controllers that Resource Manager has to track is not large, because controllers are only installed at the ingress network nodes of local [SPs.

- Topology Manager: This functional block performs the BGP routing protocol for distributing the routing information of the VPN sites, as has been already described in the introduction of the example.

- Operation Manager: The Operation Manager is responsible for determining which accounting controllers will be downloaded to each of the network nodes. Then, this component configures the AMCs Manager.

- Request Manager: The Request Manager receives requests for a VPN creation. Upon receiving the request, it checks if this VPN can be created. For example, it checks for the existence of the VPN sites of the customer.

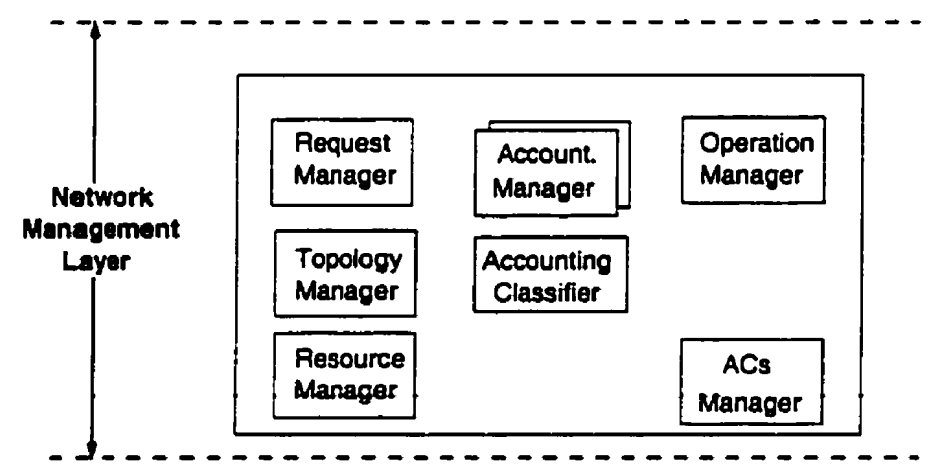

Figure 5.7: Functional Blocks in the Network Management Layer

In the Resource Management Layer the functional components are (see Figure 5.8).

- Auction-based Scheduler: When the network node is congested, then this scheduler sends traps to the Bidding Controllers of the customers at specific intervals. Furthermore, it executes the algorithm "Auction" in Figure 5.5 at each interval. According to the computed marginal cost, the scheduler forwards the flows whose bids are greater than the marginal 
cost, and all the other flows are buffered. Note that there is a big difference between this auctioning mechanism and the traditional mechanism: the bids of flows are not constant and can be changed in each auctioning interval and along the path. Finally, in Provider B, there is not an auction-based Scheduler, but a simple scheduler.

- Accounting Group Controller: The Accounting Group Controller is responsible for (1) configuring the Accounting Group in the MIB, (2) updating the MIB. This controller should configure the MIB such that the MIBlet Controllers of the customers can access particular rows in the MIB. A MIBlet Controller of a customer can access only the rows that refer to his sessions. That means that different MIBlet Controllers are permitted to read different rows/tables of MIB. Moreover, the Accounting Group of the MIB is updated by the Accounting Group Controller.

In our example, the Accounting Group Controller should meter the number of packets per traffic type of each customer, and update the appropriate ruws of the MIB. Note that, if a customer requests a detailed bill, then the MIB should be configured to keep more rows, according to the level of the granularity that customer has requested. Furthermore. customers should be aware of the space and time parameters $(s, t)$. Thus, the rows in the MIB which hold these parameters are configured by the Accounting Group Controller to be accessible by all MIBlet Controllers. Finally, the MIB in Provider $A$ has an extra row which maintains the marginal cost for each auctioning interval. Similarly, this row can be accessed by all MIBlet Controllers of customers.

- Controllers of the Customer: In our example, the customer downloads the following controllers: MIBlet controller in the network nodes of Provider $A$ and $B$, and Bidding Controllers in network nodes of Provider A (where auctioning takes place). The Bidding Controller executes the algorithm in Figure 5.4 when it receives traps from the Scheduler. The variables that should be known by the Bidding Controller are maintained in the MIBlet Controller.

The MIBlet Controller has access to specific rows of the MIB, and also can maintain its own MIB. For example, a customer can request a basic accounting summary from his provider, and maintain his own MIB. Thus, the customer can configure the Accounting Group of his MIB, as he desires. For example, he can classify his packets according to the 
applications to which they belong, and also he can maintain some statistics about his traffic.

- Classifier: This component classifies the packets according to their owner. As ve have already mentioned, owners are distinguished by the first- and second-level labels. Then it forwards the packets to the appropriate Marker.

- Marker: Each customer has the freedom to mark his packets with a class of service (realtime class or non real-time class). This is accomplished by having his own Marker. Furthermore, this component notifies the MIBlet controller, in order to update his MIBlet.

- Topology Controller: When the Topology Controller receives a message from a VPN site for a VPN route creation, it assigns a Label Switch Path (LSP) for the traffic originating from this VPN site. More specifically, it assigns a distinct VPN-IP address to this site of the VPN, and attaches the appropriate community attribute to the route used by the BGP protecol. Another responsibility of the Topology Controller is to label the unlabeled packets coming into the network node.

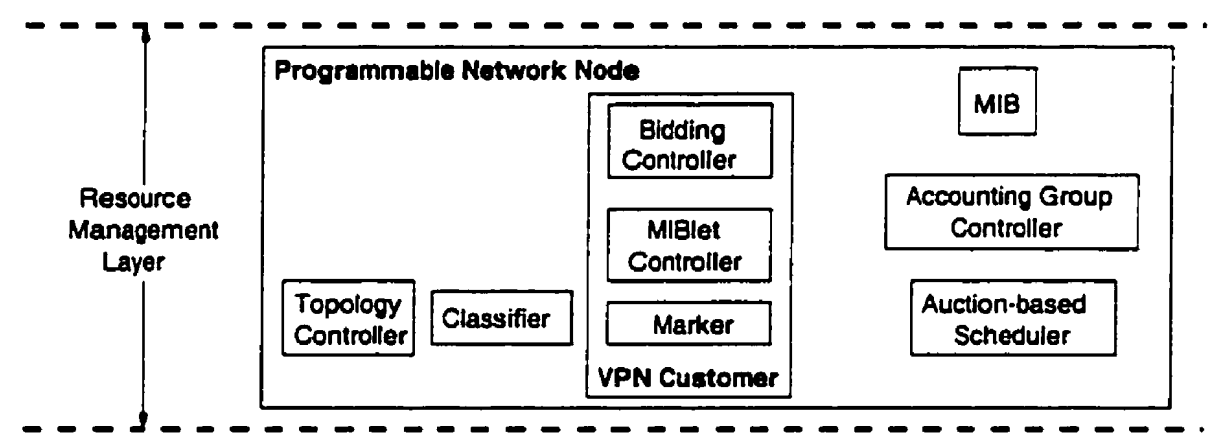

Figure 5.8: Functional blocks in the Resource Management Layer of Provider A. In Provider B the Bidding Controller does not exist and instead of having an auction-based scheduler. there is a simple scheduler

\subsubsection{Messages Exchanged among the Functional Blocks}

In this section, first, we describe the protocol that is used for the creation of a VPN. Then, we explain the path that is followed by the packets inside a network node. Finally, we describe an accounting protocol, which includes the messages that are exchanged among the accounting mechanisms.

The customer requests VPN services from the two VPN providers. The messages that are 
exchanged among the customer and either of VPN providers are exactly the same. Specifically, the steps for VPN creation are (see Figure 5.9):

1. The Operation Manager of the customer sends a VPN creation request to the Request Manager of Provider $A$ and to the Request Manager of Provider $B$. The VPN creation request includes also the location of the customer's sites and their local ISPs.

2. The Request Managers of both providers ( $A$ and $B$ ) check if the VPN can be created. If it is feasible, then they instantiate an Accounting Manager for this new customer to perform the accounting management functions.

3. The Request Managers also send a message to their Topology Managers in order to notify them of the creation of a new VPN.

4. The Request Managers send a response to the Operation Manager of the customer to indicate that an Accounting Manager was created. This response includes: (1) a port number of the Accounting Manager, which will be used by the Accounting Manager of the customer in order to exchange accounting information; (2) the IP addresses of the ingress provider routers. If the VPN creation is not feasible, then the Operation Manager of the corresponding provider notifies the customer that his request cannot be satisfied (i.e., sends a negative notification).

5. The Operation Manager of the customer creates an Accounting Manager, and forwards these two port numbers to his Accounting Manager. However, if Operation Manager has received a negative notification by any provider, then it quits.

6. The Accounting Manager of the customer establishes a direct connection with each of the two Accounting Managers of Provider A and B.

7 . The Operation Manager of the customer sends a request to his Topology Manager for instantiating the VPN route creation. (This request includes the IP addresses of the ingress provider routers.)

8. The Topology Manager of the customer sends these IP addresses to his edge routers.

9. The Customer's edge routers send a request for creating the paths that will connect the customer's sites.

10. The Topology Controllers associate a Label Switch Path between the provider edge routers; and then inform the Accounting Group Controller of the new VPN Customer; the Accounting 
Group Controller creates a new row in the MIB for keeping the customer's accounting information.

11. The Topology Controllers inform the Classifiers of the new VPN Customer; Classifiers according to the first-and second-level labels forward the packets to the appropriate controllers.

12. The Topology Controllers attach a community to the VPN site and send the route to the Topology Managers (which execute the BGP protocol).

13. When Topology Managers finish the distribution of the VPN route, they send a message to the Request Manager of their providers, in order to notify them that the route was created for this customer successfully.

14. The Request Managers of the two providers send a VP:N route creation response to the Operation Manager of the customer to indicate that the route of VPN was created.

15. The Operation Manager notifies the AMCs Manager to transfer the appropriate accounting controllers (AMCs) to the provider routers.

16. The AMCs Manager transfers the same MIBlet Controller, and Marker to the edge routers. Furthermore, it downloads the Bidding Controller (which actually executes the algorithm in Figure 5.4) to the edge router of Provider A.

Packets coming into a network node pass through the following components:

1. Topology controller, which labels the unlabeled packets coming into the network node and then forwards them, to the Classifier.

2. Classifier, which classifies each packet according to the labeis and sends them to the corresponding Marker.

3. Marker, which notifies the corresponding MIBlet Controller for this packet, and then forwards them to the Scheduler.

4. Scheduler, which notifies the Accounting Group Controller for the packets that pass through, and then forwards them to the output link

The accounting protocol for charging a VPN customer takes place both in the Resource Management Layer and in Network Management Layer with different time granularities. At the lower layer, charging and pricing is performed on the order of minutes or seconds, whereas at the higher 


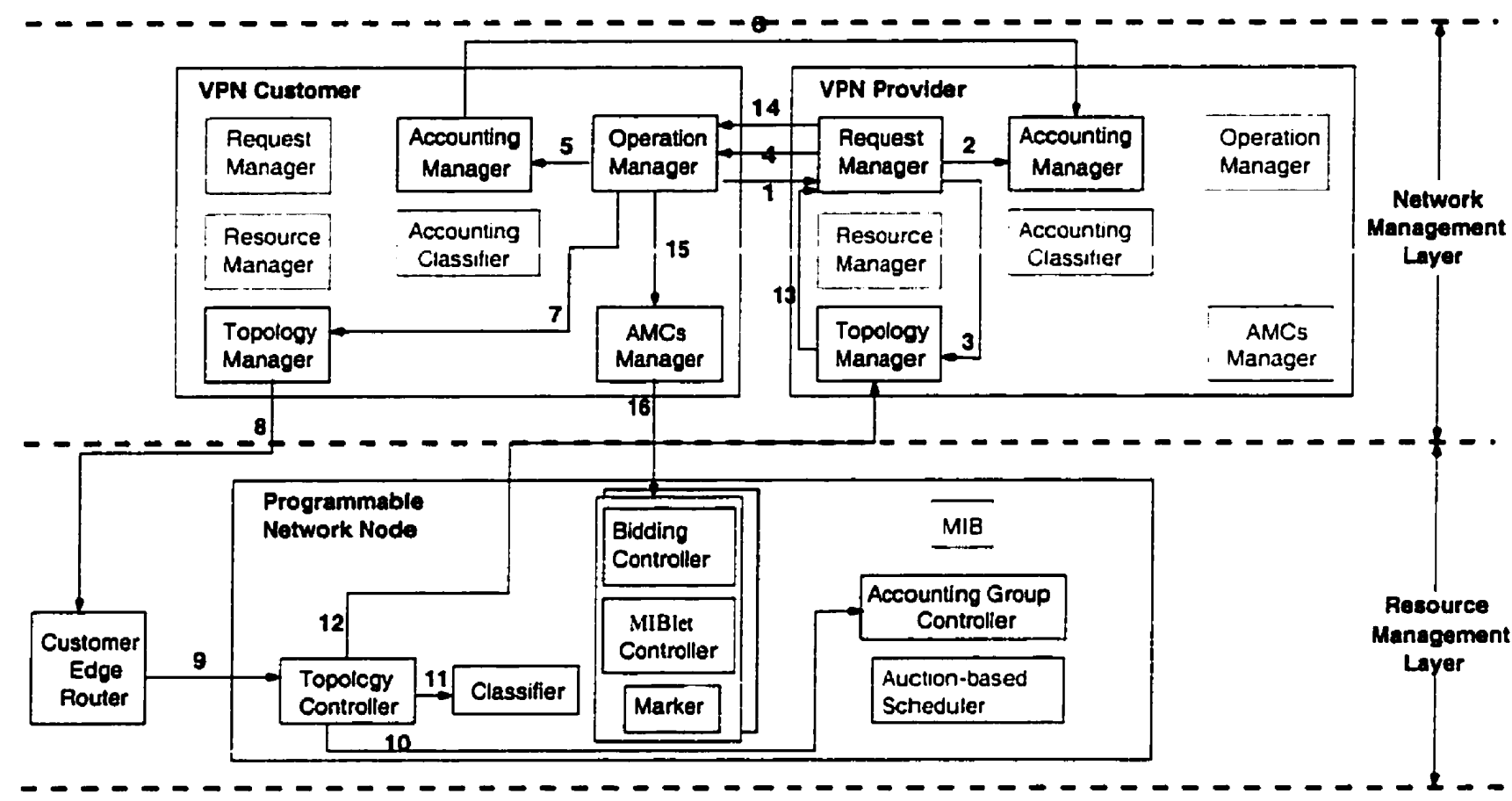

Figure 5.9: Creating a VPN

layer these two processes are performed on the order of hours or months. In our scenario, we have charging and pricing processes at the network nodes only for Provider $A$.

Thus, the accounting protocol in the Resource Management Layer of Provider $A$ is (see Figure 5.10):

1. The Auction-based Scheduler sends traps to the Bidding Controllers of its customers at specific intervals (called auctioning intervals) when there is congestion.

2. Bidding Controllers post their bids to the Auction-Based Scheduler (i.e., the algorithm in Figure 5.4).

3. The Auction-based Scheduler performs the algorithm shown in Figure 5.5; the Scheduler informs the Accounting Group Controller of the new marginal cost, in order to charge correctly the customers whose packets are transmitted to the output link.

4. The Accounting Group Controller updates the appropriate row in the MIB with the new marginal cost.

On the contrary, the Provider B sells the notwork resources on long time scales. Thus, the 


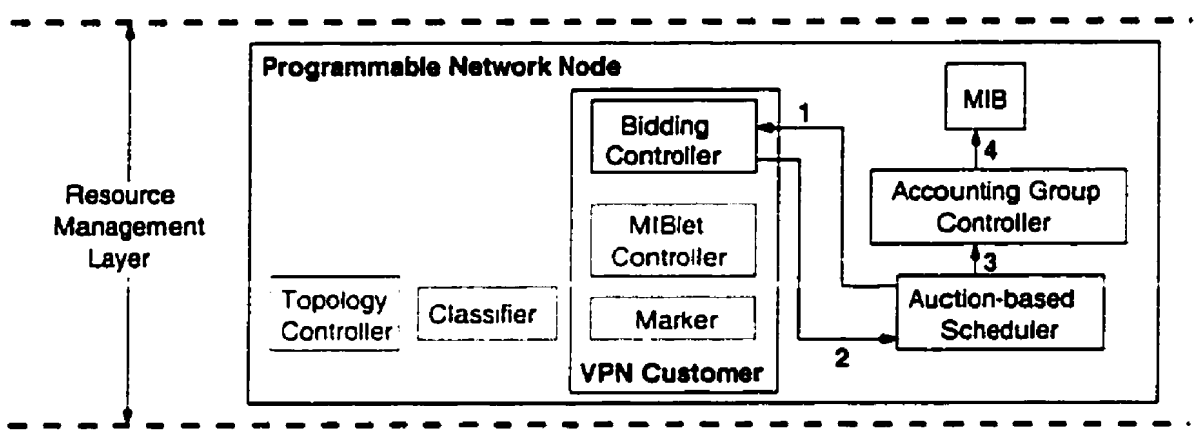

Figure 5.10: Charging and Pricing in the Resource Management Layer of Provider A

charging and pricing are executed only in the Network Management Layer. The messages that are exchanged between the Accounting Manager of Provider B and the Accounting Manager of customers just after the VPN creation are:

1. The Accounting Manager in Provider B sells the SLAs to the new customer (i.e., it performs the function "Selling SLAs" of Figure 5.2).

2. The Accounting Manager of customer selects the SLAs, and informs the Provider B of which SLAs it selected (i.e., the function "Buying SLAs" of Figure $\mathbf{5 . 2}$ is performed).

3. The Accounting Manager of the customer informs its Resource Manager of the SLAs it bought from Provider $B$.

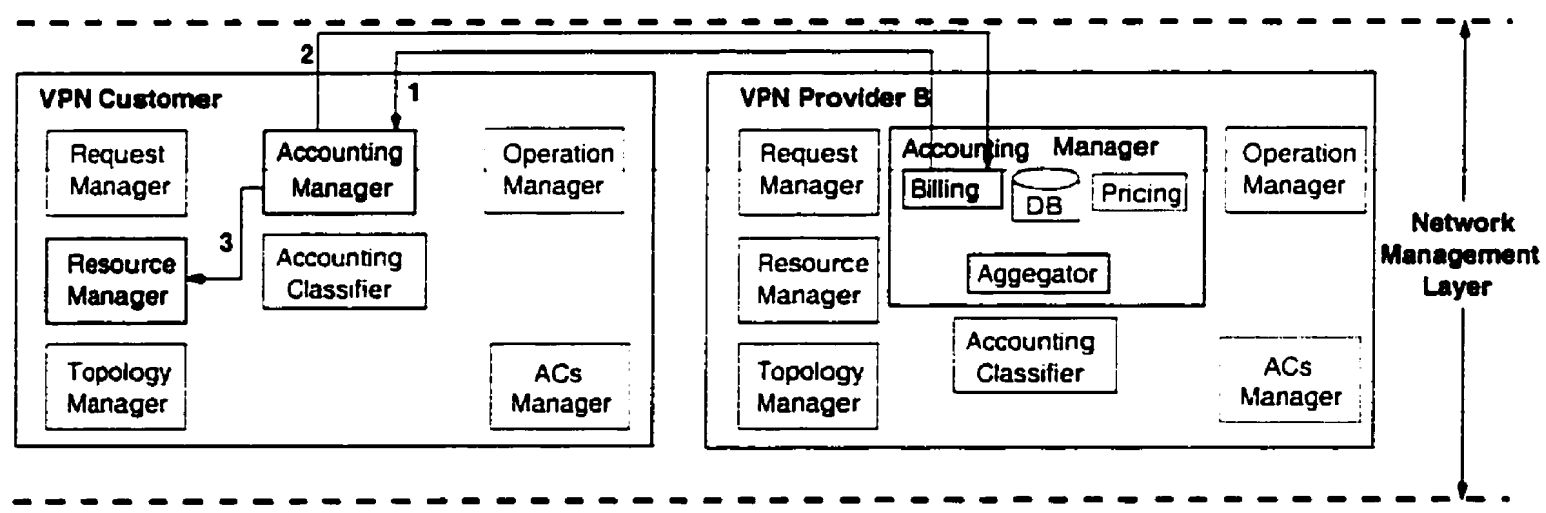

Figure 5.11: Charging and Pricing in the Network Management Layer of Provider B

Finally, the steps for collecting the metering data in Provider B and in Provider A are (see Figure 5.12): 
1. Whenever the Accounting Group Controller in a network node has enough metering data to transmit, then it transmits them to the Accounting Classifier of his provider.

2. Classifier forwards the accounting data to the appropriate Accounting Manager. Classification is done according to the customer to whom the accounting data belongs, i.e., according to the first- and second-level labels.

3. The Aggregator of Accounting Manager receives the accounting data, and updates the Accounting Database.

4. The Billing retrieves the accounting status of a VPN Customer from the Accounting Database.

5. The Billing issues analytical invoices, and also supports real-time accounting by sending the current status of the accounting record of a VPN customer at specific time intervals. The invoice is sent to the Accounting Manager of the customer.

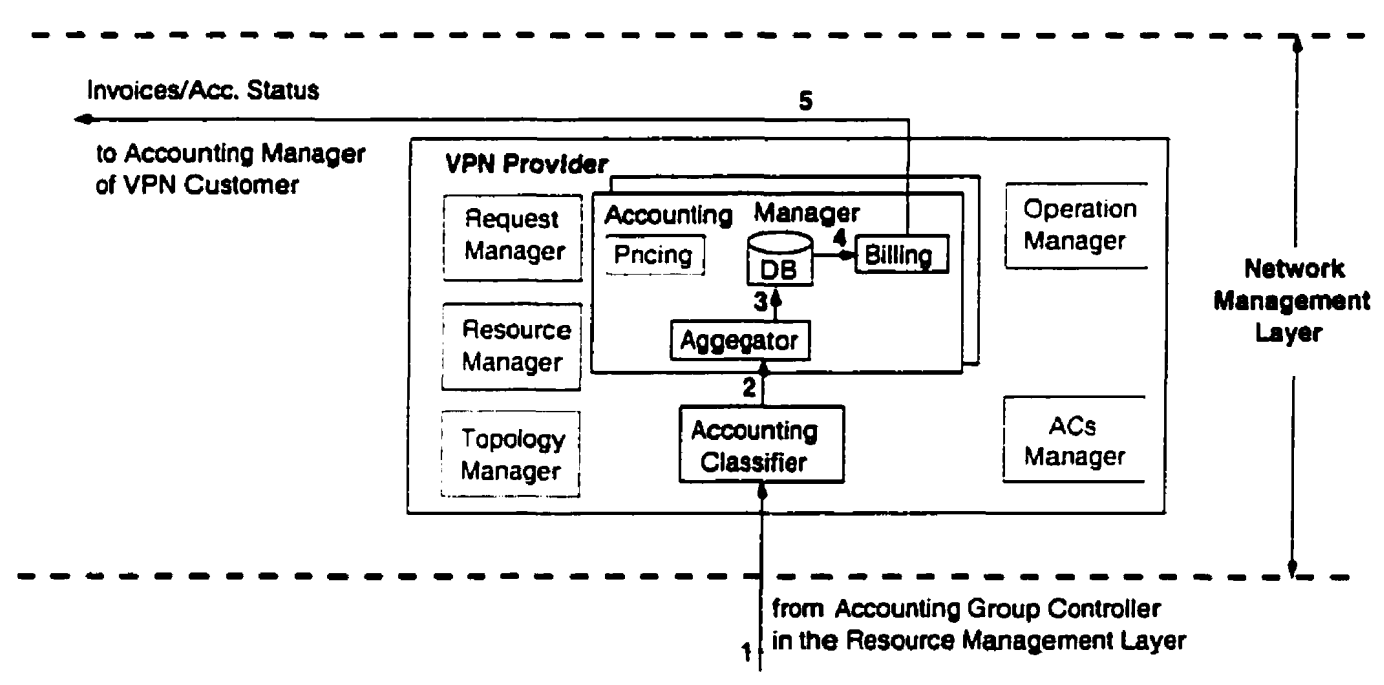

Figure 5.12: Charging the VPN Customer

\subsection{Further Extensions of the Proposed Example}

Let us further extend the proposed example and add some intelligent accounting tasks:

- The customer wants to execute a "Smart Marker" in the network nodes of Provider B. "Smart Marker" monitors the network load, and whenever there are free resources in the non real-time class of service, it marks his real-time traffic as non real-time. Note, that 
he will not experience any performance penalty, because the non real-time class of service is under-utilized. In this way, the customer saves money, since the non real-time class is cheaper than the real-time class of service.

- The customer wants to check whether there are errors in the bills or not. Therefore, the customer meters his traffic using his own MIBlet controller, periodically. Then he computes his expenses and compares them with the bills received by the provider. This has the advantage that providers are under customer surveillance, and they cannot "cheat" them.

- Providers wholesale their network resources, and in turn the VPN customer retails the network resources. Users of the VPN do not have to deal with the heterogeneous pricing policies of ISPs, because they pay only the VPN customer. In addition, the VPN customer employs pricing models which can be easily understood by its users. To be more specific, pricing models which are closer to the category of flat-rates attract more users, because their expenses can be foreseen. This scenario is feasible in our architecture because the VPN customer has the flexibility to monitor, and maintain his own MIIB through the MIBlet controller; and to mark the packets by applying his own rules.

- A provider wants to charge the customers for the CPLi cycles consumed by their controllers, in order to discourage them from executing heavyweight controllers. Thus, the provider just downloads a controller to the network node, which monitors the CPU and maintains a MIB. This MIB contains a table with the CPU cycles that have been wasted by each customer. Finally, the provider includes in the bill of each customer the cost of the CPU.

- A provider decides to make the accounting protocol more efficient at the expense of requiring more CPU cycles in the network node. Thus, he executes a controller which compresses the accounting data before transmitting them to the Accounting Mangers.

\subsection{Advantages}

The contributions of our architecture to the realization of the above scenario are fundamental. First, the smart-market (i.e., auction) pricing model is employed without introducing a new protocol. Bidding controllers are executed in the network and make this pricing model feasible. Second, customers can exploit the weakness of the non real-time pricing model, by performing the "smart-marker". Thus, customers increase their surplus. Third, the customer's desire to modify 
his bid for specific packets can be achieved through the bidding controllers. However, if the bid was carried at the header of the packets, then the customer could not change the bid to react to network state. Fourth, real-time pricing models can be applied without any delay, because everything is executed inside the network node, where the data exist. Fifth, the power of MIBlet controllers give the freedom to customers to retail their network resources. They can maintain their own MIBs, and they can also organize their accounting data as they desire. Sixth, a provider can make the accounting protocol more efficient by executing a controller which compresses the accounting data. Finally, accounting management is more flexible, economically efficient, and incurs less network overhead due to the programmability of network nodes. 


\section{Chapter 6}

\section{Conclusions}

In this section, we compare our architecture with other accounting architectures, and then we present the future work of this thesis. Finally, we summarize our work.

\subsection{Related Work}

Our work was inspired by the work of Travostino [1], in which the AIACE (Active IP Accounting Co-processor Environment) is proposed. AIACE considers active networks for accounting. It dynamically pushes the intelligence into network nodes and exploits the power of open and active network nodes for accounting. Our architecture is fully compatible with the AIACE. However, we exploit the programmability of network nodes for charging and pricing mechanisms in addition to accounting. Furthermore, the programmable end-points can also perform accounting management tasks. Finally, we propose a specific accounting management architecture for programmable VPNs.

Briscoe et al. propose an architecture that follows the principle that pricing, charging, metering and accounting should be performed at the end-points [39]. He introduces the term active tariffs. meaning that prices are estimated at the end-points according to the congestion signals from the network nodes, and also according to the tariffs of network nodes. In the literature, there have already been proposed real-time pricing schemes which execute at the end-points (such as by Marbach [29]). In Section 4.5, we claimed that there are circumstances where accounting management at the end points is not preferable. Therefore, in our architecture, we support accounting management in both the end-points and the network nodes.

Gunther et al. describe an architecture of QoS-enabled VPNs over the Internet [44]. This work is a part of the CATI project funded by the Swiss National Science Foundation. DiffServs are supported by having service brokers for communication between different domains as well as 
within domains. Customers agree on SLAs, which must be flexible enough to store a vast variety of payment schemes. These SLAs can be established dynamically. In order to provide QoS through the entire network. SLAs should be negotiated between the customer and the ISP, and also among the ISPs across the communication path. The main differences from our architecture is that they do not deal with real-time pricing models and they do not consider the programmable networks for providing VPN services. Thus, their architecture is not as flexible and configurable as ours. Furthermore, they do not address the issue of setting the prices. Similar to our architecture, they have also adopted the edge-pricing model, in which the VPN customer is charged only by the local ISPs. Finally, they define specific SLAs, which can also be deployed in our architecture at the network management layer.

In the following paragraphs, we outline some architectures for accounting in the Internet. These architectures are orthogonal to our architecture (i.e., policy vs. mechanism), because open interfaces are not considered and specific policies are defined. Moreover, our architecture has been proposed in the context of programmable VPNs, whereas the following architectures have been proposed for providing general IP services. However, the Accounting Management Controllers can be deployed for charging IP services, and they are not restricted only to the VPN services.

In SUSIE [45] (an ACTS project), they proposed a charging and accounting architecture for IntServ, DiffServ and ATM. They adopted charging schemes for ATM services and they transferred them to the IntServ and DiffServ. Furthermore, the mapping of the services in the IntServ architecture into the ATM services has been examined from the charging perspective. Finally, the implementation of this architecture was based on the TINA architecture. They have implemented several charging approaches, but there is not any result about the effectiveness and fairness of each approach.

To our knowledge, there are only two projects which have investigated the efficiency of usagebased pricing through experiments. The Internet Demand Experiment (INDEX [25]) is a prototype alternative ISP model that offers differentiated-quality service on demand, with prices that reflect resource cost. The findings of INDEX project are that today's system of flat-rate pricing by ISPs is very inefficient, and also ISPs with differentiated quality of service is technically feasible. Moreover, they have shown that customers pay less and suppliers increase profits. Another implementation of a network billing system was for the New Zealand Gateway [18], where users were charged by volume for their traffic, in order to cover the costs of this gateway. They have developed the 
NeTraMet [19].

Edell et al. describe a system for billing users for their traffic [46]. The main characteristic of this system is that users are charged instead of hosts (i.e., the IP address of the hosts). This is accomplished by postponing the establishment of connections while the user is contacted, verifying in a secure way that he is prepared to pay. Their results show that this billing system is feasible even for large campus networks. Furthermore, they indicate that pricing schemes may be used to control network congestion.

In a radical work, Braun et al. discuss two basic principles in accounting and billing management [ $[t]$ ]: (l) decentralized accounting where a backbone service provider charges its adjacent service providers and in turn these providers charge their adjacent providers, etc. and (2) multiple service queues.

Apostolopoulos provides a preliminary work in which the author introduces the principle of open accounting technology that can be used to build a proprietary billing scheme [-18]. This is achieved with private MIBs for each individual operator. The private MIBs proposed in this work, are similar to the MIBlet controllers used in our architecture. These MIBs also belong to the users of network node and they let users control their traffic characteristics and have access to their accounting information. Finally, he proposed the Temporal MIB which represents the past and the current accounting information. The main difference from our architecture is that this architecture did not consider programmable network nodes, and thus the private MIBs are as rigid as the traditional MIB.

\subsection{Future Work}

Future work of this thesis involves the following issues:

- Bandwidth broker: Both the VPN subscriber and the VPN provider should deploy bandwidth brokers. The role of bandwidth broker is to control and coordinate the resource allocation. The functionality of the bandwidth broker in the VPN subscriber is more complex than the one in the VPN provider. Specifically, the provider's bandwidth broker has only to negotiate SLAs with the customers and check if there are free resources for the new customer. On the other hand, the subscriber's broker has to buy SLAs from more than one ISP, and it has to coordinate the resources bought from each ISP. The major concern of this bandwidth broker is to buy an equal amount of resources from each of the ISPs; otherwise, 
the subscriber wastes the resources bought from one of its ISPs. In the example presented in the previous chapter, a bandwidth broker is deployed in the Network Management Layer. As we have already mentioned, the Resource Manager of the subscriber operates as a bandwidth broker. Moreover, the Billing component on the provider's side acts as the bandwidth broker.

- Extending the current open interfaces to an open accounting interface: Another item of future work of this thesis involves implementation issues. Specifically, the interfaces which have been proposed for the programmable networks should be extended in order that the user be able to execute tasks for accounting management. The open accounting interface should give the flexibility to the user to modify any field of his packet, and should let the users apply low-level monitoring.

\subsection{Summary}

This thesis has addressed the issue of accounting management in the context of programmable networks. It shows that programmable networks can enhance every operation of accounting management, i.e., metering, accounting, charging, pricing, and billing. These operations are discussed from the architectural perspective, and we do not propose a specific accounting management application.

Common problems of packet-level charging and pricing schemes can be overcome. if we use programmable networks. We push all the mechanisms into the network nodes from the end-points. Hence, no pricing policies are transmitted, and the traffic overhead is reduced. Furthermore, there is no delay, because the mechanisms retrieve their parameters at the same site where they are performed. Another important benefit is that these mechanisms can be executed more than one time along the path. Specifically, they can be executed where there is congestion, and users are able to select a class of service according to the network load in the network core. This is not facilitated by programmable networks. Finally, we show these advantages in a specific example.

In this thesis, we proposed an accounting management architecture for programmable VPNs. We exploit MIBlets [37] in order to isolate the accounting information of various VPNs which share the same network resources. Another feature of our architecture is that we let the accounting management tasks be performed both in the hosts and in the network nodes. The decision depends on many parameters discussed in this thesis. 
Finally, an example with specific accounting management tasks is presented. Through this example, the advantages of programmable networks supported by accounting management are shown. 


\section{Bibliography}

[1] Franco Travostino. Towards an Active IP Accounting Infrastructure. IEEE OPE.VARCH, 2000.

[2] Andrew T. Campbell. Herman G. De Meer. Michael E. Kounavis, Kazuho .Miki, John B. Vicente, and Daniel Villela. A Survey of Programmable Networks. AC.M SIGCO.M.M Computer Communication Review. 1999.

[3] R. Braden, D. Clark, and S. Shenker. Integrated Services in the Internet Architecture: an Overview. RFC 1633, 1994. http://www.ietf.org/rfc.html.

[4] J. Wroclawski. Specification of the Controlled-Load Network Element Service. RFC 2211. 1997. http://www.ietf.org/rfc.html.

[5] S. Shenker, C. Partridge, and R. Guerin. Specification of Guaranteed Quality of Service. RFC 2212, 1997, http://www.ietf.org/rfc.html.

[6] S. Blake, D. Black, M. Carison, E. Davies. Z. Wang, and W. Weiss. An Architecture for Differentiated Services. RFC 2475, 1998. http://www.ietf.org/rfc.html.

[7] V. Jacobson, K. Nichols, and K. Poduri. An Expedited Forwarding PHB. RFC 2598, 1999. http://www.ietf.org/rfc.html.

[8] J. Heinanen, Telia Finland, F. Baker, W. Weiss, and J. Wroclawski. Assured Forwarding PHB Group. RFC 2597, 1999. http://www.ietf.org/rfc.html.

[9] Kazem Najafi. A Novel Cost Model for Active Networks. Proc. of Int. Conf. on Communication Technologies, World Computer Congress 2000, August.

[10] Opensig Working Group. http://www.comet.columbia.edu/opensig/. 
[11] David L. Tennenhouse, Jonathan M. Smith, W. David Sincoskie, David J. Wetherall, and Gary J. Minden. A Survey of Active Network Research. IEEE Communications Magazine, $35(1): 80-86$, January 1997 .

[12] Paul Ferguson and Geoff Huston. What is a VPN? White Paper. 1998.

[13] Rosen E. and Rekhter Y. BGP/MPLS VPNs. RFC 2547, 1999. http://www.ietf.org/rfc.html.

[14] Andrew Do-Sung Jun and A. Leon-Garcia. Virtual Network Resources Management: A Divide-and-Conquer Approach for the Control of Future Networks. IEEE Crlobal Telecommunications Conference, 1998.

[15] The Genesis Project. http://comet.ctr.columbia.edu/genesis/.

[16] S. Shenker, D. Clark. D. Estrin, and S. Herzog. Pricing in Computer Networks: Reshaping the Research Agenda. AC.M Computer Communication Review, Vol. 26. No. 2, 1996.

[17] David D. Clark. A Model for Cost Allocation and Pricing in the Internet. MIT Workshop in Internet Economics, 1995.

[18] Nevil Brownlee. New Zealand Experiences with Network Traffic Charging. .IIT Workshop in Internet Economics, 1995.

[19] Nevil Brownlee. NeTrailet Version 4.3 Now Available.

[20] N. Brownlee, C. Mills, and G. Ruth. Traffic Flow Measurement: Architecture. RFC 2063, 1997. http://www.ietf.org/rfc.html.

[21] N. Brownlee. Traffic Flow Measurement: Meter MIB. RFC 2064, 1997. http://www.ietf.org/rfc.html.

[22] David Mitton, Michael St.Johns, Stuart Barkley, Dave Nelson, Basavaraj Patil, Mark Stevens, and Barney Wolff. Introduction to Accounting Management. Internet Draft, January 2001. http://www.ietf.org/[D.html.

[23] J. Mackie-Mason and Hal Varian. Pricing the Internet. Public Access to the Internet. Brian Kahin and James Keller, eds. Cambridge, MA: MIT Press, 1995. 
[24] Luiz A. DaSilva. Pricing for QoS-Enabled Networks: A Survey. IEEE Communications Survey and Tutorials, Second Quarter 2000.

[25] Richard Edell and Pravin Varaiya. Providing Internet Access: What We Learn From INDEX. IEEE, Neturork '1.3, n.5, Sept-Oct 1999.

[26] Matthias Falkner, Michael Devetsikiotis, and loannis Lambadaris. An Overview of Pricing Concepts for Broadband IP Networks. IEEE Communications Survey and Tutorials. Second Quarter 2000.

[27] A. M. Odlyzko. Paris Metro Pricing: The Minimalist Differentiated Services Solution. IEEE Proceedings Seventh International Workshop on Quality of Service (IWQoS '99), pages 159$161,1999$.

[28] Alok Gupta, Date O. Stahl, and Andrew B. Whinston. A Priority Pricing Approach to Manage Multi-Service Class Networks in Real-Time. MIT Workshop in Internet Economics. 1995.

[29] P. Marbach. Differentiated Services Networks: Pricing and Software Agents. Technical Report CSRG-422, 2001.

[30] Jeffrey $k$. Mackie-Mason. A Smart-Market for Resource Reservation in a Multiple Quality of Service Information Network. 1997.

[31] Jeffrey K. Mackie-Mason, Liam Murphy, and John Murphy. The Role of Responsive Pricing in the Internet. MIT Workshop in Internet Economics, 1995.

[32] Frank P. Kelly. Charging and Accounting for Bursty Connections. WIT Workshop in Internet Economics, 1995.

[33] Costas Courcoubetis and Vasilios A. Siris. Managing and Pricing Service Level Agreements for Differentiated Services. 6th IEEE/IFIP International Conference of Quality of Service (IWQoS'99), 1999.

[34] Berbard Aboba, Jari Arkko, and David Harrington. Introduction to Accounting Management. RFC 2975, October 2000. http://www.ietf.org/ID.html. 
[35] Ingo Busse. Accounting Management for Global Broadband Connectivity Services. IEEE, Network Operations and Management Symposium, 1998.

[36] Aiko Pras, Bert-Jan van Beijnum, Ron Sprenkels, and Robert Parhonyi. Internet Accounting. IEEE Communications Magazine, May 2001.

[37] Walfrey Ng, Andrew Do-Sung Jun, Hungkei Keith Chow, Raouf Boutaba, and Alberto LeonGarcia. MIBlets: A Practical Approach to Virtual Network Management. Sirth IFIP/IEEE International Symposium on Integrated Network Management, 1999.

[38] K. Ramakrishnan and S. Floyd. A Proposal to Add Explicit Congestion Notification (EC:N) to IP. RFC 2\{81, 1999. http://www.ietf.org/rfc.html.

[39] Bob Briscoe, Mikc Rizzo, Jerome Tassel, and Konstantinos Damianakis. Lightweight Policing and Charging for Packet Networks. IEEE OPENARCH, 2000.

[40] George Fankhauser and Bernhard Plattner. DiffServ Bandwidth Brokers as Mini-Markets. Workshop on Internet Service Quality Economics, .WIT, Cambridge, 1999.

[41] Bruce Davie and Yakov Rekhter. MPLS:Technology and Applications. Morgan Kaufmann Publishers, 2000.

[42] F. P. Kelly. On Tariffs. Policing and Admission Control for . Iultiservice Networks. 15th International Teletraffic Congress (ITC - 15), 1996.

[43] C. Courcoubetis, F. P. Kelly, V. A. Siris, and R. Weber. A Study of Simple Usage-Based Charging Schemes for Broadband in the Internet. IFIP International Conference on Broadband Communications (BC'gs).

[44] Manuel Gunter, Torsten Braun, and Ibrahim Khalil. An Architecture for Managing QoSEnabled VPNs over the Internet. IEEE, Local Computer Networks, 1999.

[45] SUSIE Project (ACTS). , 1999. http://www.teltec.dcu.ie/susie/.

[46] Richard J. Edell, Nick McKeown, and Pravin P. Varaiya. Billing Users and Pricing for TCP. IEEE Journal On Selected Areas In Communication, 13(7), September 1995. 
[4i]] Hans-Werner Braun, Kimberly C. Claffy, and George C. Plyzos. A Framework for Flow-based Accounting on the Internet. IEEE, International Conference on Information Engineering, 1993.

[48] Theodore K. Apostolopoulos. Accounting Management in Communications Networks: Concepts and Architecture. IEEE. Computers and Communications, 1997. 